\title{
Hsp90ab1 stabilizes LRP5 to promote epithelial-mesenchymal transition via activating of AKT and Wnt/ $\beta$-catenin signaling pathways in gastric cancer progression
}

\author{
Huanan Wang ${ }^{1} \cdot$ Guangxu Deng ${ }^{1} \cdot$ Meiling Ai ${ }^{2,3} \cdot{\text { Zhijun Xu } \mathrm{Xu}^{1} \cdot \text { Tingyu } \mathrm{Mou}^{1} \cdot \text { Jiang } \mathrm{Yu}^{1} \cdot \mathrm{Hao} \mathrm{Liu}^{1} \cdot \text { Shuang Wang }}^{2,4}$. \\ Guoxin $\mathrm{Li}^{1}$
}

Received: 2 November 2017 / Revised: 29 August 2018 / Accepted: 10 September 2018 / Published online: 10 October 2018

(c) The Author(s) 2018. This article is published with open access

\begin{abstract}
Hsp90ab1 is upregulated in numerous solid tumors, which is thought to induce the angiogenesis and promote cancer metastasis. However, it's actions in gastric cancer (GC) has not been exhibited. In this study, Hsp90ab1 was demonstrated to be overexpressed and correlated with the poor prognosis, proliferation and invasion of GC. Ectopic expression of Hsp90ab1 promoted the proliferation and metastasis of GC cells both in vitro in cell line models of GC and in vivo using two different xenograft mouse models, while opposite effects were observed in Hsp90ab1 silenced cells. Moreover, the underlining molecular mechanism was explored by the co-immunoprecipitation, immunofluorescence, GST pull-down and in vitro ubiquitination assay. Namely, Hsp90ab1 exerted these functions via the interaction of LRP5 and inhibited ubiquitinmediated degradation of LRP5, an indispensable coreceptor of the Wnt/ $\beta$-catenin signaling pathway. In addition, the crosstalk between Hsp90ab1 and LRP5 contributed to the upregulation of multiple mesenchymal markers, which are also targets of Wnt/ $\beta$-catenin. Collectively, this study uncovers the details of the Hsp90ab1-LRP5 axis, providing novel insights into the role and mechanism of invasion and metastasis in GC.
\end{abstract}

These authors contributed equally: Huanan Wang, Guangxu Deng, Meiling Ai

Electronic supplementary material The online version of this article (https://doi.org/10.1038/s41388-018-0532-5) contains supplementary material, which is available to authorized users.
Shuang Wang
shuangw@126.com
$\triangle$ Guoxin Li
gzliguoxin@163.com

1 Department of General Surgery, Nanfang Hospital, Southern Medical University, Guangdong Provincial Engineering Technology Research Center of Minimally Invasive Surgery, Guangzhou 510515, China

2 Department of Pathology, Southern Medical University, Nanfang Hospital, Guangzhou 510515, China

3 Department of Radiotherapy, Affiliated Cancer Hospital \& Institute of Guangzhou Medical University, Guangzhou 510515, China

4 Department of Pathology, School of Basic Medical Sciences, Southern Medical University, Guangzhou 510515, China

\section{Introduction}

Gastric cancer (GC) is the fifth most common cancer globally and the third most common cause of cancer-based deaths in both males and females [1]. In China, GC ranks second among cancer deaths, and the incidence of GC is continually increasing [2-4]. Despite advances in both the diagnosis and therapy for GC in the past decade, GC survival has not markedly improved. Clinical evidence has demonstrated that the early development and dissemination of micro-metastatic cells may be responsible for tumor relapse and metastasis [5, 6]. However, little is known about the exact molecular mechanisms responsible for GC metastasis.

Accumulating evidence has shown that there is a close relation between the epithelial-mesenchymal transition (EMT) and cancer metastasis [7-9]. Multiple signaling pathways have been reported to be involved in EMT, including the AKT/mTOR, Wnt and AMPK pathways [1014]. For example, prior researches have demonstrated that in non-small cell lung cancer and GC, tumor cells exhibit loss of the adhesion molecule biomarker E-cadherin and acquire the expression of mesenchymal biomarkers 
A
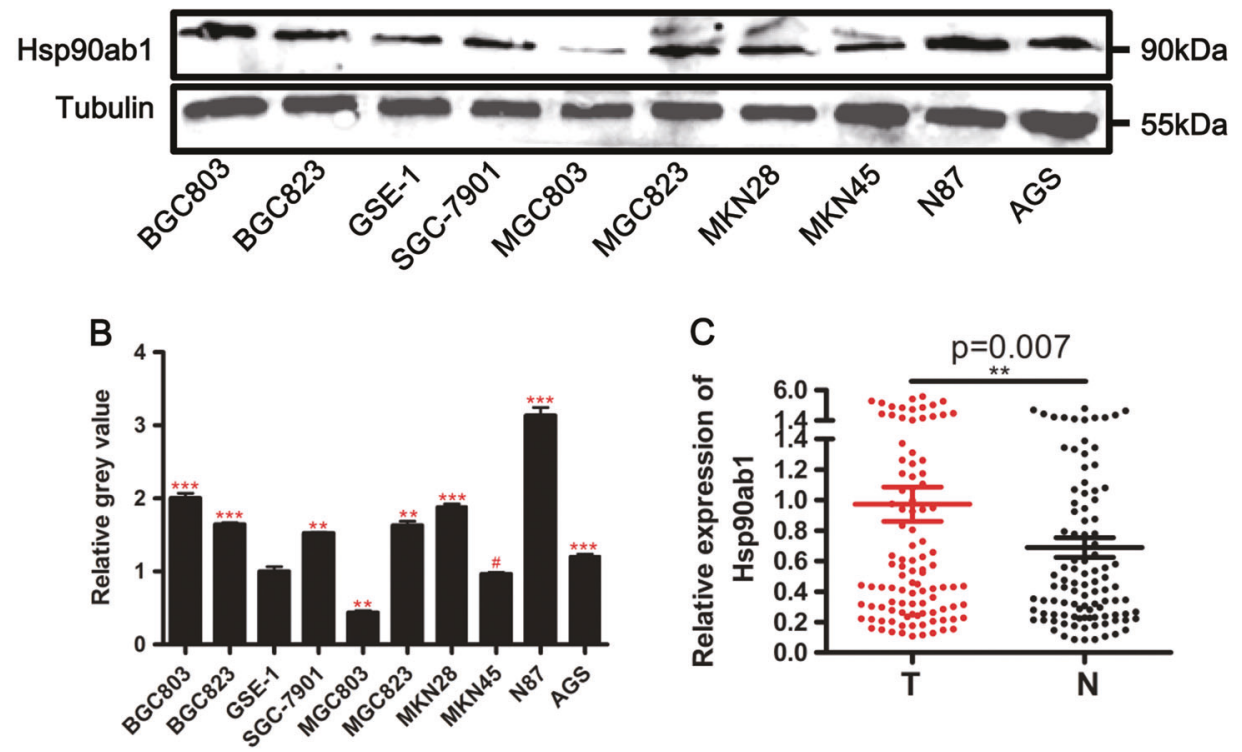

D
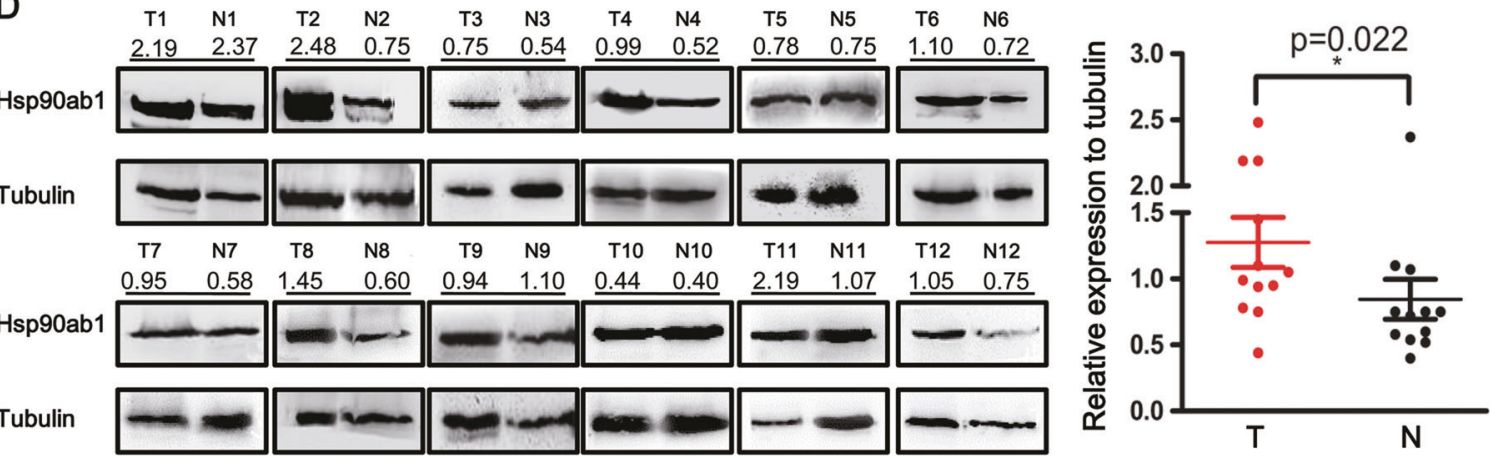

Fig. 1 Hsp90ab1 expression was up-regulated in GC cell lines and patients. a Expression levels of Hsp90ab1 were determined by Western blotting in GC and gastric mucosa epithelial cell lines. b The relative protein expression levels were quantified by using Quantity One Software, and the relative protein abundance of Hsp90ab1 in the individual cells was calculated by normalization with Tubulin expression. c Expression of Hsp90ab1 in 102 paired human GC tissues

by q-PCR, which was determined by normalization with GAPDH control. d Expression analyses of Hsp90ab1 protein in the 12 paired gastric carcinomas samples by Western blotting. $N$ normal mucosa, $T$ tumor. The protein expression levels were quantified by Quantity One Software, and the relative protein abundance was determined by normalization with Tubulin. Error bars represented the mean \pm SD of three replicates. \#p $>0.05 ; * p<0.05 ; * * p<0.01 ; * * * p<0.001$

Vimentin or N-cadherin, proteins involved in the EMT, by the activation of TGF- $\beta$ or Wnt $/ \beta$-catenin $[13,15]$. Therefore, we must explore key molecules involved in the invasion and metastasis, which may provide new insights into therapeutic targets.

The heat shock proteins are among the most abundantly expressed proteins in mammalian cells, and have previously been reported to have a role in the regulation of the tumorigenesis [16-20]. The heat shock proteins form a multiprotein chaperone complex which mediated the correct folding and stabilization of substrates involved in the cell cycle, proliferation, migration, and apoptosis [20-23]. In mammalian cells, there are four isoforms of Hsp90: Hsp90aa1, Hsp90ab1, GRP94, and TRAP1 [24], and
Hsp90aa1 is the most well-studied one among them [25]. A recent study showed that Hsp90 inhibition prevented from proper folding and stabilization of its substrates, which resulted in the ubiquitination and degradation of the clients by the proteasome pathway [18].

Previous researches have demonstrated that Hsp90 promotes tumorigenesis in GC, breast cancer, non-small cell lung cancer, hepatocellular carcinoma, and conjunctival melanoma [24, 26-28]. Hsp90 overexpression has been associated with decreased survival in these cancer patients as well [29]. Hsp90 may promote tumorigenesis in part due to its increased affinity for ATP and ATPase activity in cancer cells [16-20]. It is also reported that Hsp90ab1 overexpression promotes the angiogenesis, metastasis and 


\section{A Score 0}

Score 1

Score 2

Score 3

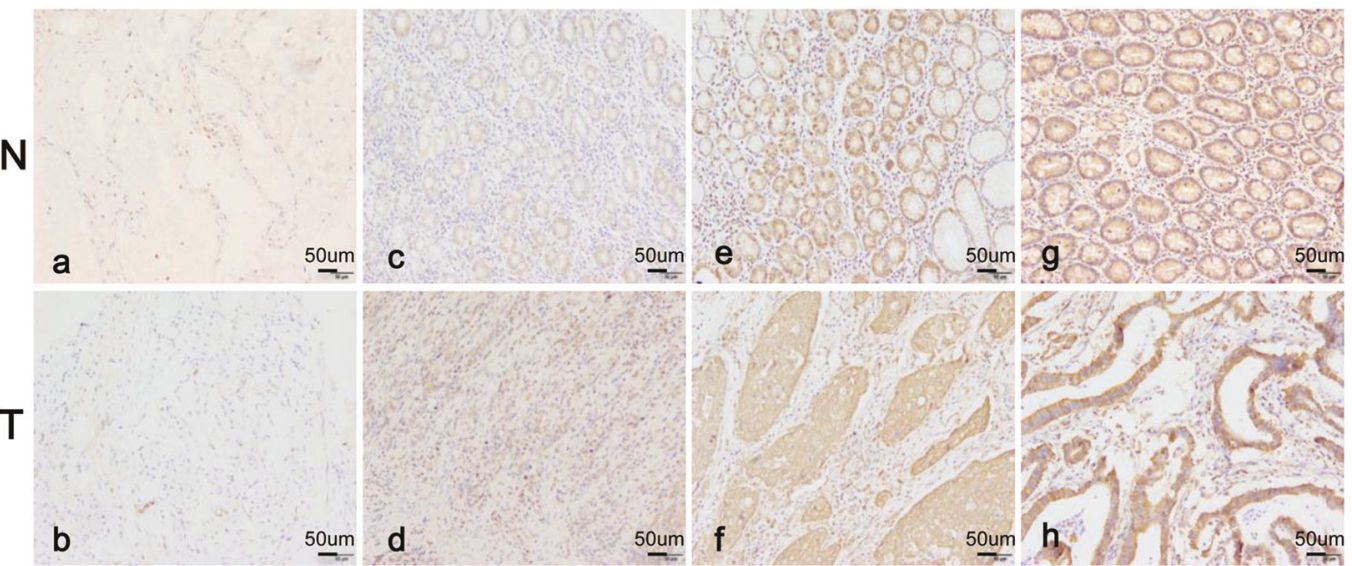

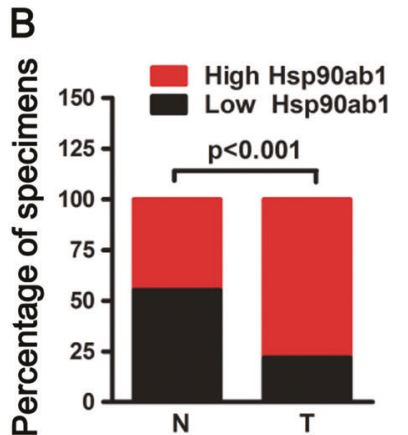

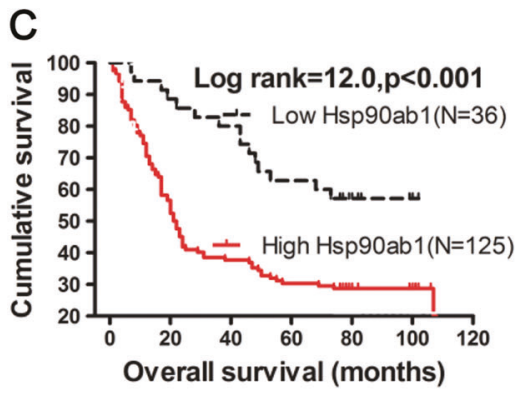

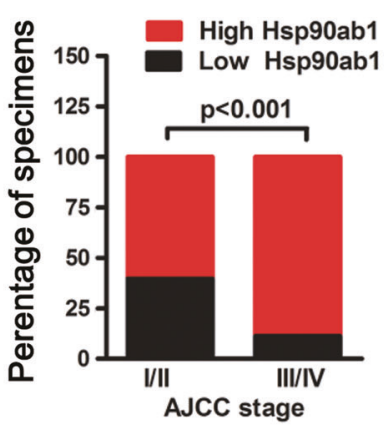

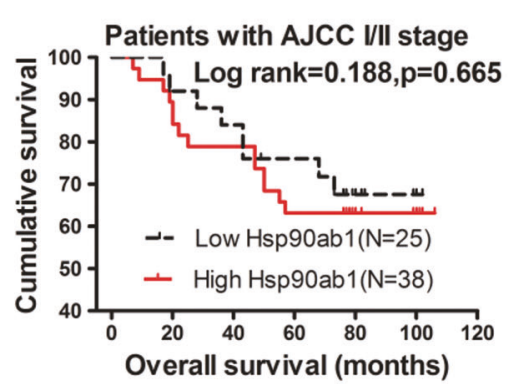

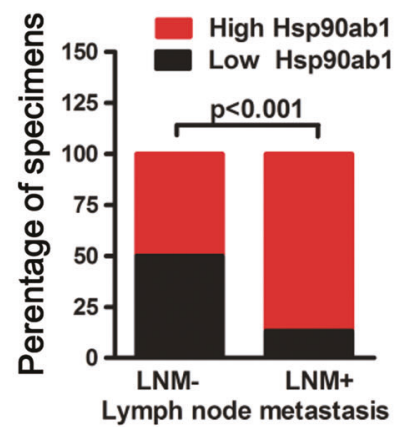

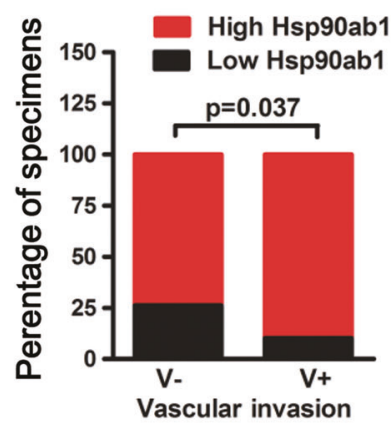

Fig. 2 Hsp90ab1 expression was positively associated with progression and poor prognosis and could be an independent prognostic factor for GC patients. a IHC analysis of Hsp90ab1 expression in GC tissue samples $(\mathrm{T})$ and surrounding non-tumor tissues $(\mathrm{N})$. The representative figures of GC tissues with low and high Hsp90ab1 levels of the staining intensity were shown. b Graphical illustration of statistical Hsp90ab1 distribution in GC tissues. Hsp90ab1 was significantly higher in GC than their adjacent nontumorous tissues. Hsp90ab1 protein expression was more frequently found in GC with AJCC classification (III/IV) than that with AJCC classification (I/II). Also Hsp90ab1 protein expression was more frequently found in GC with lymph node metastasis, vascular invasion than that in GC without lymph node metastasis, vascular invasion. c Kaplan-Meier survival analysis for GC patients with distinct expression level of Hsp90ab1 and AJCC classification after surgical resection differentiation of hepatocellular carcinomas and lung cancer $[24,30]$. In other tumors, numerous studies have demonstrated that Hsp90aal participates in tumorigenesis. However, the role of Hsp90ab1 in GC carcinogenesis has not been elucidated comprehensively so far.

Here, we hypothesized that Hsp90ab1 promotes GC metastasis, leading to a worse prognosis, by activation of the EMT. We began by investigating the relationship of
Hsp90ab1 with patient survival. Subsequently, in order to better understand the mechanisms of underlying GC progression, we used GC cell lines and mouse models to elucidate the role of aberrant Hsp90ab1 expression in GC tumorigenesis. Finally, we confirmed that Hsp90ab1 stabilized LRP5 to promote EMT via activating the AKT and $\mathrm{Wnt} / \beta$-catenin signaling pathways. 
Table1 Univariate and multivariate analysis of different prognostic parameters for GC patients

\begin{tabular}{|c|c|c|}
\hline Variables & $\mathrm{HR}(95 \% \mathrm{CI})$ & $P$ value \\
\hline \multicolumn{3}{|l|}{ Univariate analysis } \\
\hline Gender $(\mathrm{M} \text { vs } \mathrm{F})^{\mathrm{a}}$ & $1.440(0.975-2.128)$ & 0.067 \\
\hline Age $($ years $)(<60$ vs $\geq 60)$ & $0.955(0.643-1.418)$ & 0.818 \\
\hline Location $(\mathrm{C} / \mathrm{B} \text { vs } \mathrm{A} / \mathrm{W})^{\mathrm{b}}$ & $0.826(0.556-1.228)$ & 0.345 \\
\hline Tumor size $(\mathrm{cm}$ in diameter $)(>5.5 \mathrm{vs} \leq 5.5)$ & $1.707(1.127-1.945)$ & 0.007 \\
\hline Vascular invasion (yes vs no) & $1.481(0.671-2.155)$ & 0.005 \\
\hline Histological stage $(\mathrm{P} / \mathrm{U} \text { vs } \mathrm{W} / \mathrm{M})^{\mathrm{c}}$ & $2.121(1.259-3.573)$ & 0.005 \\
\hline AJCC stage (I/II vs III/IV) & $4.559(2.845-7.306)$ & $<0.001$ \\
\hline $\mathrm{T}$ stage (T1/T2 vs T3/T4) & $3.632(1.761-7.492)$ & $<0.001$ \\
\hline $\mathrm{N}$ stage (N0 vs N1 3) & $3.992(2.224-7.166)$ & $<0.001$ \\
\hline M stage (M0 vs M1) & $2.613(1.379-4.952)$ & 0.003 \\
\hline Hsp90ab1 expression (low vs high) & $2.525(1.457-4.377)$ & 0.001 \\
\hline \multicolumn{3}{|l|}{ Multivariate analysis } \\
\hline AJCC stage (I/II vs III/IV) & $4.166(2.573-6.746)$ & $<0.001$ \\
\hline Vascular invasion (yes vs no) & $1.524(1.177-1.974)$ & 0.001 \\
\hline Hsp90ab1 expression (low vs high) & $1.983(1.130-3.478)$ & 0.017 \\
\hline \multicolumn{3}{|l|}{$H R$ hazard ratio, $C I$ confidence interval } \\
\hline \multicolumn{3}{|l|}{$*$ Statistically significant $(P<0.05)$} \\
\hline \multicolumn{3}{|l|}{${ }^{\mathrm{a}} M$ male, $F$ female } \\
\hline${ }^{\mathrm{b}} C$ cardia, $B$ body, $A$ antrum, $W$ whole & & \\
\hline
\end{tabular}

\section{Results}

\section{Up-regulation of Hsp90ab1 in GC tissues correlates with GC metastasis}

We originally compared the expression of Hsp90ab1 mRNA and protein in a panel of GC cell lines to gastric mucosa epithelial cell line (GSE-1). Seven out of nine GC cells had increased Hsp90ab1 protein expression (Fig. 1a, b) to varying degrees. Additionally, six GC cell lines showed higher mRNA expression level of Hsp90ab1 compared to GSE-1 cells (Figure S1A). To confirm whether Hsp90ab1 was also upregulated in GC, Hsp90ab1 mRNA and protein expression was assessed in patient samples of GC tissues and adjacent normal gastric mucosa tissues removed during radical resection. The level of Hsp90ab1 mRNA was dramatically upregulated in the $102 \mathrm{GC}$ tissues compared with their paired normal samples (Fig. 1c, Figure S1B, $P<0.01$ ). In addition, in the 12 sample pairs, Hsp90ab1 protein expression was significantly higher in the GC tissues than that in the normal gastric samples (Fig. 1d, $P<0.05$ ). Finally, we performed IHC analysis of a tissue microarray. Hsp90ab1 expression was up-regulated in 135 cases and downregulated in the other 38 cases in the GC tissue samples, whereas the positive rate of Hsp90ab1 was significantly low in the normal gastric mucosa (Fig. 2a, b, $\chi^{2}=$ 49.237, $P<0.001)$. Taken together, these results indicated that Hsp90ab1 was up-regulated in GC.
To explore whether Hsp90ab1 expression level was related to GC progression, we analyzed the association between Hsp90ab1 and clinicopathologic status in 161 GC patients with complete pathological and follow-up data. Hsp90ab1 protein expression level was positively associated with lymph node metastasis (Fig. $2 b, \chi^{2}=23.421, P$ $<0.001$ ), vascular invasion (Fig. $2 \mathrm{~b}, \chi^{2}=4.343, P<0.05$ ), American Joint Committee on Cancer (AJCC) stage (Fig. $\left.2 \mathrm{~b}, \chi^{2}=17.889, P<0.001\right)$ and $\mathrm{T}$ stage (Table S3, $\chi^{2}=$ 6.313, $P<0.05$ ). However, no significant correlation was found between Hsp90ab1 protein expression and other clinicopathological parameters (Table S3). Furthermore, overall survival of 161 patients with follow-up data displayed that higher Hsp90ab1 expression was significantly correlated with reduced overall survival in GC patients (Fig. 2c, log-rank test $=12.0, P<0.001$ ), which was supported by public data from Kaplan-Meier plotter using Gene Expression Omnibus and TCGA datasets (Figure S1C-D) (KM plotter, http://kmplot.com)[31, 32]. Furthermore, multivariate survival analysis suggested that Hsp90ab1 expression $[P=0.017 ; \mathrm{HR}=1.983 ; 95 \%$ confidence interval (CI), 1.130-3.478], AJCC stage $(P<0.001 ; \mathrm{HR}=$ 4.166; 95\% CI, 2.573-6.746) and vascular invasion $(P=$ $0.001 ; \mathrm{HR}=1.524 ; 95 \% \mathrm{CI}, 1.177-1.974)$ were confirmed to be independent prognosis factors of disease outcome in GC patients (Table 1). Overall, these findings strongly suggested that Hsp90ab1 expression was associated with GC invasion and migration. 
Fig. 3 Silencing of Hsp90ab1 inhibited cell proliferation, invasion, and migration in vitro. a Hsp90ab1 mRNA in BGC823 and MKN28 cells after shRNAmediated knockdown of Hsp90ab1 was detected by realtime RT-PCR. b-d Downregulation of Hsp90ab1 resulted in inhibiting the proliferation and DNA replication of BGC823 and MKN28, as detected by colony formation assays (b), EdU incorporation assay (c) and CCK-8 assays (d). e The invasion ability of GC cells was significantly reduced in Hsp90ab1-depleted cells compared with the control groups, as revealed by Matrigel invasion assays. $\mathbf{f}$ Downregulation of Hsp90ab1 reduced the migration ability of GC cells, as determined by Scratchwound-healing assays. The data was expressed as the mean $\pm \mathrm{SD}$ and reproduced in three independent experiments. $* P<$ $0.05 ; * * P<0.01 ; * * * P<0.001$
A
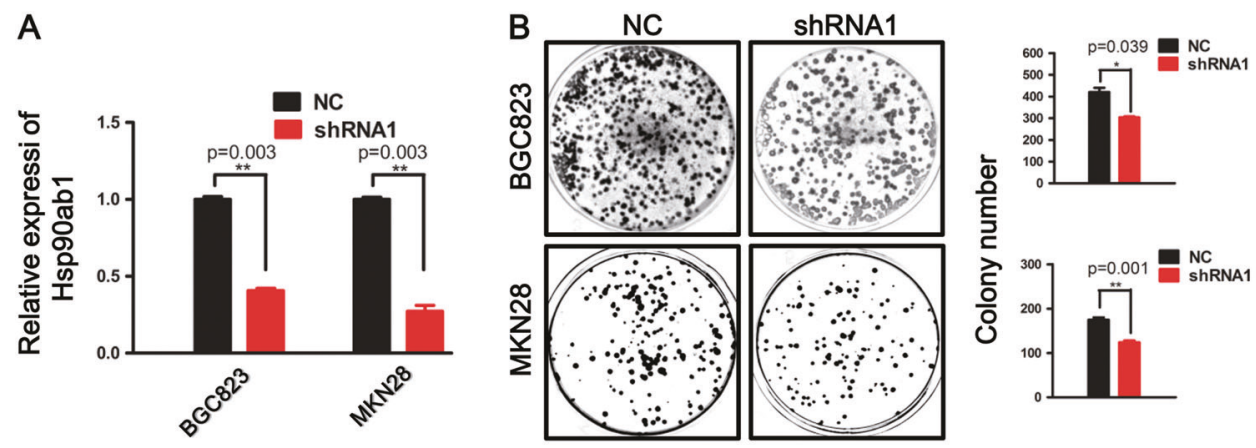

C

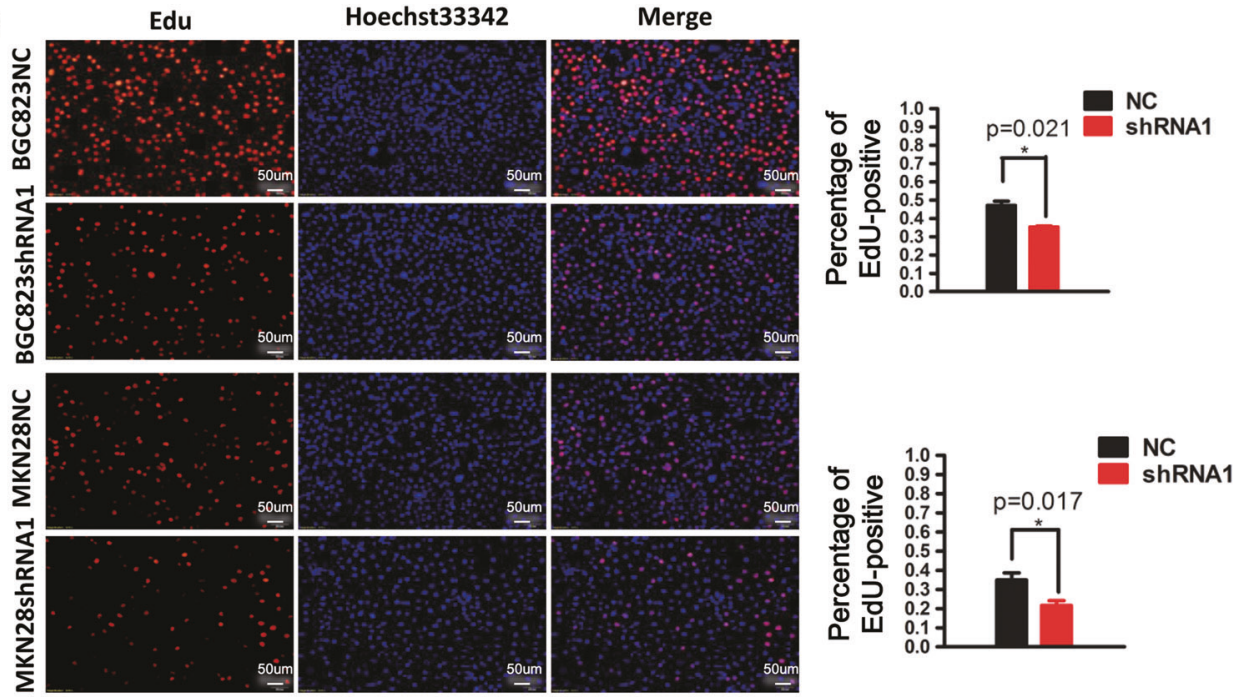

D
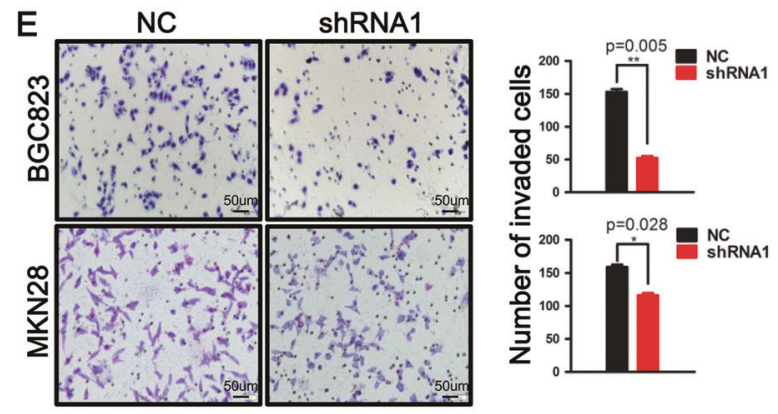

F
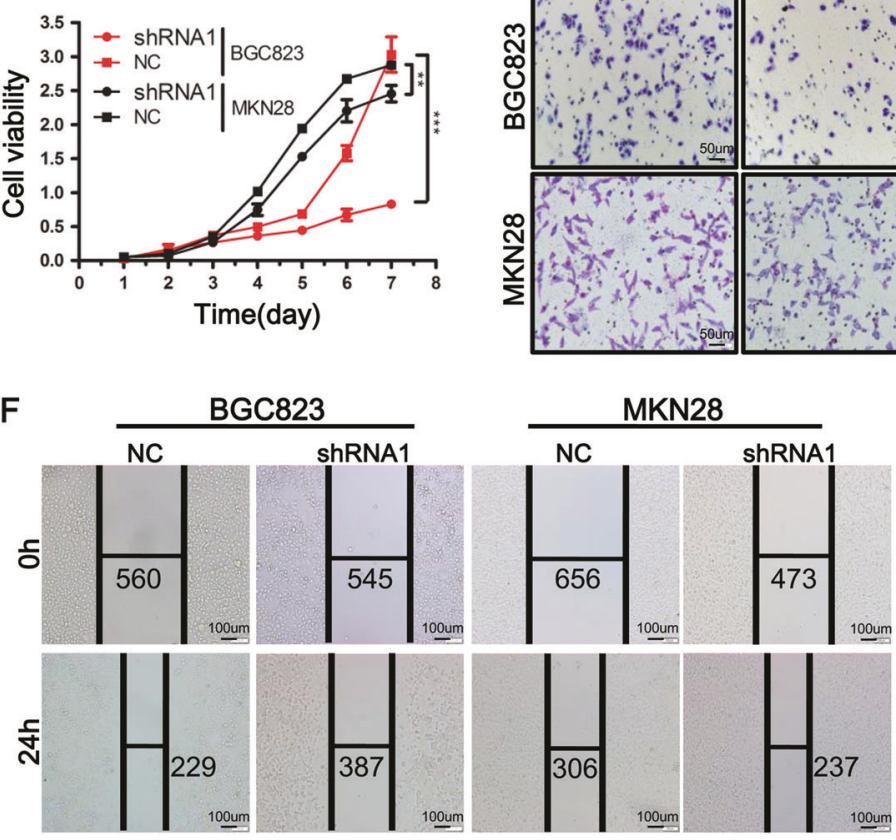

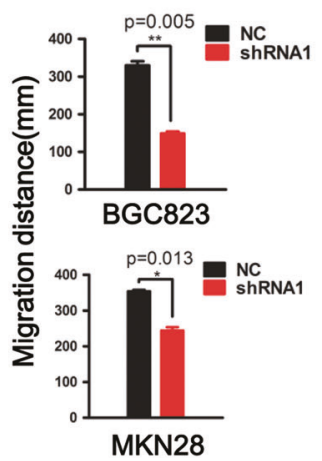


A

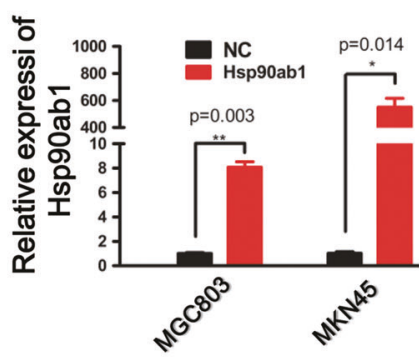

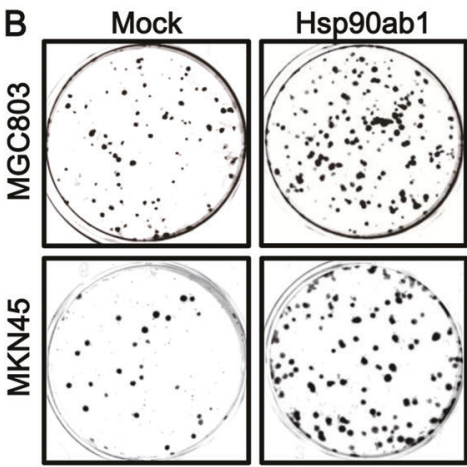

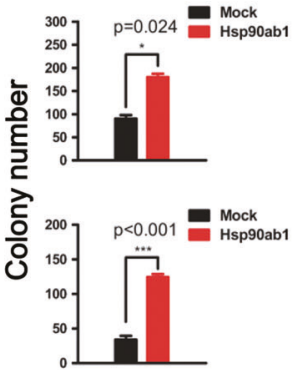

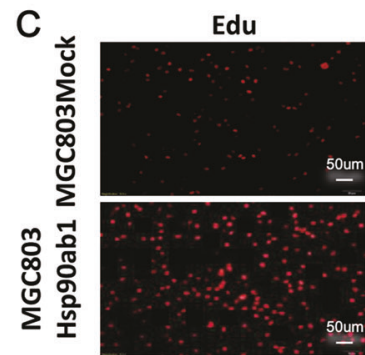

Hoechst33342
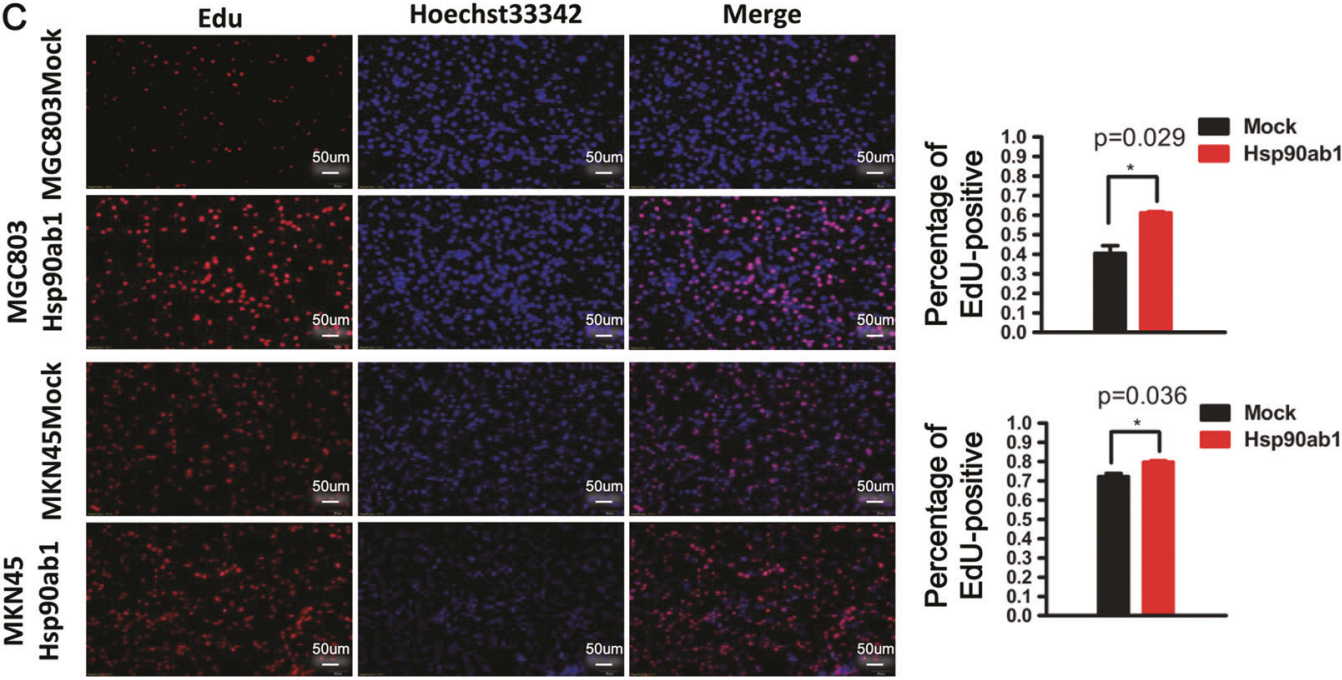

D

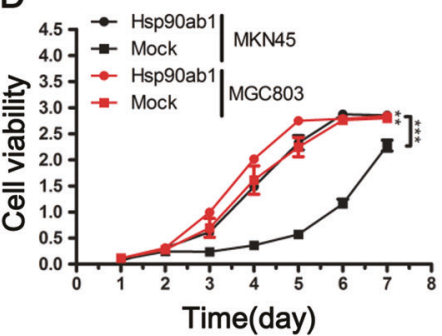

G $\quad \mathrm{IC} 50(\mu \mathrm{M}) 95 \% \mathrm{Cl}$ $35.8433 .26-38.61$ - MGC803-Hsp90ab1 86.32 80.40-92.68

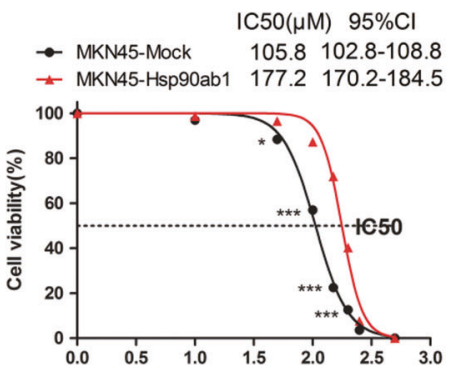

E Mock Hsp90ab1
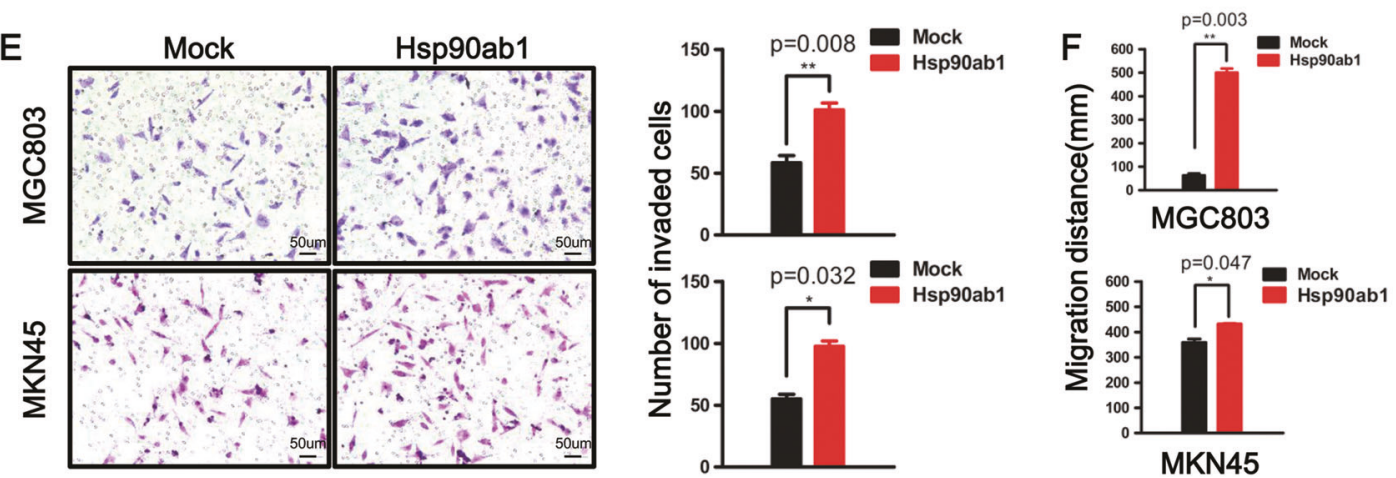
Fig. 4 Overexpression of Hsp90ab1 promoted cell proliferation, invasion and migration and tolerance to chemotherapeutic drugs in vitro. a Expression analyses of Hsp90ab1 mRNA in MGC803 and MKN45 cells after lentivirus-mediated overexpression of Hsp90ab1. b-d Elevated expression of Hsp90ab1 induced significantly higher growth rates, DNA replication and proliferation of MGC803 and MKN45 in GC cells. e The invasion ablity of GC cells was enhanced in Hsp90ab1-overexpressing cells compared with the control groups. $\mathbf{f}$ Up-regulation of Hsp90ab1 promoted the migration ability of GC cells. g Growth inhibitory effect of oxaliplatin on Hsp90ab1overexpressing and empty vector-transfected MGC803 and MKN45 cells. The data was expressed as the mean $\pm \mathrm{SD}$ and reproduced in three independent experiments. $* P<0.05 ; * * P<0.01 ; * * * P<0.001$

\section{Knockdown of Hsp90ab1 represses GC cell proliferation, invasion, and migration in vitro}

To verify if Hsp90ab1 is necessary for GC oncogenesis, endogenous Hsp90ab1 expression was silenced in BGC823 and MKN28 cells. qRT-PCR analysis proved a significant decrease of Hsp90ab1 expression in the shRNA1 group compared with the control group (Fig. $3 \mathrm{a}, P<0.01$ ). The capacity to form colonies in the Hsp90ab1-depleted cells was remarkably suppressed compared with the cells transfected with control vectors (Fig. $3 b, P<0.05$ ). Similarly, we measured cell proliferation visualizing the incorporation of EdU into DNA. Depletion of Hsp90ab1 significantly reduced cell proliferation as compared to controls (Fig. 3c, $P<0.05)$. Finally, the CCK- 8 assay revealed that the downregulation of Hsp90ab1 could markedly inhibit GC cell growth compared with the negative control (Fig. 3d, $P$ $<0.01$ ). We also performed colony formation assays, CCK8 cell proliferation assays and EdU staining with shRNA2 in BGC823 and MKN28 cell lines, respectively. Our data were consistent with our previous results (Figs. S2B-2D, $P$ $<0.05)$. Finally, the knockdown of Hsp90ab1 in GC cells significantly reduced both cell invasion and migration capacity, as illustrated by Matrigel invasion assay and wound healing assay (Fig. 3e, f, Figure S2E, $P<0.05$ ). These data strongly suggested that Hsp90ab1 was essential for the invasion and migration of GC cells.

Besides, to validate whether the Hsp90ab1-depletion suppresses progression through cell cycle, cellular DNA content were quantified by flow cytometry. The depletion of Hsp90ab1 caused a significant reduction of DNA content, suggesting impeded $\mathrm{S}$ phase progression (Figure S3A). These results indicated that the downregulation of Hsp90ab1 inhibited the proliferation of GC cells in vitro.

\section{Ectopic overexpression of Hsp90ab1 promotes GC cell proliferation, invasion and migration and tolerance to chemotherapeutic drugs in vitro}

To show Hsp90ab1 is sufficient to promote cell proliferation and migration, we adopted gain-of-function models with MKN45 and MGC803 cells. To confirm the overexpression of Hsp90ab1, we measured mRNA levels after transfection with Hsp90ab1 lentiviral vectors. Transfected cells significantly upregulated Hsp90ab1 as compared to control cells (Fig. 4a, $P<0.01$ ). As revealed by colony formation assays, GC cells that overexpressed Hsp90ab1 had a dramatically increased growth rate as compared to mock-transfected cells, indicated by increased formation of colonies (Fig. 4b, $P<0.05$ ). Additionally, Hsp90ab1 overexpression in both cell lines resulted in increased cell proliferation as measured by an EdU incorporation assay (Fig. $4 c, P<0.05)$. Similarly, the increased cell numbers were also observed in the Hsp90ab1 overexpression group, indicated by CCK-8 (Fig. 4 d, $P<0.01$ ). Finally, the proportion of cells in $\mathrm{S}$ phase was increased and the proportion of cells in G1 phase was decreased to a comparable degree (Figure S3B). These results indicated Hsp90ab1 promoted cell growth by promoting cell cycle progression.

To determine the effects of Hsp90ab1 on cell migration and invasion, we observed the effect of Hsp90ab1 overexpression by a Matrigel invasion assays and a wound closure assay. Significantly higher migration rates and more rapid wound closures were observed in the group of Hsp90ab1-overexpressing compared with the control (Fig. 4e, f, Figure S3C). Collectively, these data demonstrated that Hsp90ab1 expression induced GC cell proliferation, invasion, and migration.

To investigate the effects of Hsp90ab1 expression on the chemosensitivity of gastric cancer cells to chemotherapeutic agents, MGC803 and MKN45 cells stably transfected with Hsp90ab1 lentiviral vectors and empty vector were treated with oxaliplatin at different concentrations for $24 \mathrm{~h}$. As revealed in Fig. 4g, Hsp90ab1 significantly increased the tolerance of MGC803 and MKN45 cells to oxaliplatin.

\section{Hsp90ab1 promotes the growth and metastasis of human GC cell in vivo}

Then we sought to demonstrate that Hsp90ab1 promotes GC development in vivo. Therefore, we generated xenograft models of GC by subcutaneously injecting GC cells stably transfected with Hsp90ab1 and empty vector into nude mice. Mice with Hsp90ab1-overexpressing cells developed significantly larger tumors than those transfected with control cells (Fig. 5a, b). The tumors that developed from Hsp90ab1-transfected GC cells weighed significantly more than the controls at the termination of the experiment (Fig. 5c, $P<0.05)$. Hematoxylin and eosin staining (H\&E) revealed that the Hsp90ab1-transfected tumors had similar histopathological features to human GC samples (Fig. 5d). To quantify cell growth in the tumor samples, we visualized Hsp90ab1 and Ki-67 expression in paraffin-embedded xenograft tumors. In concordance with the findings 
A

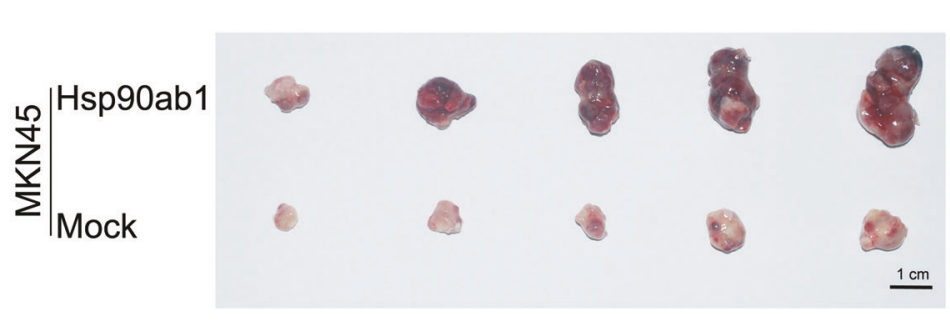

$\mathrm{B}$

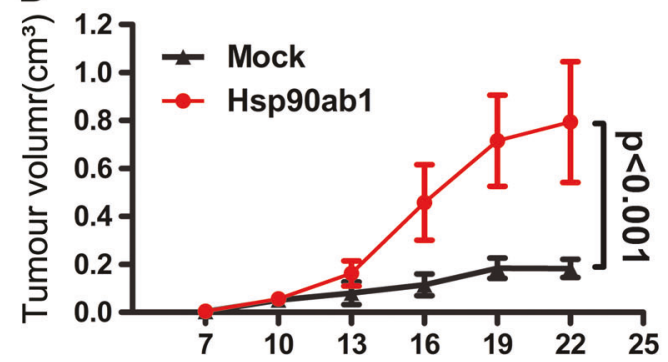

D

HE

Hsp90ab1

Ki-67

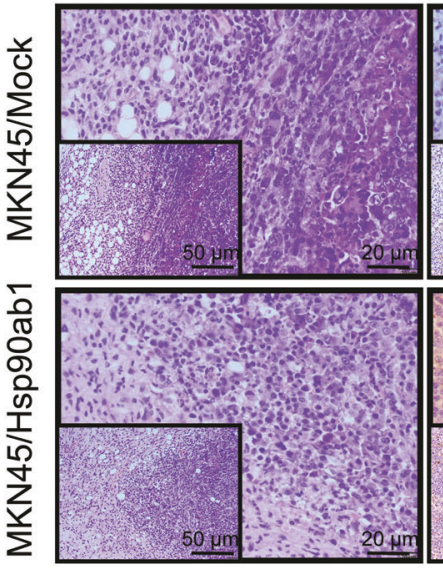

G

E

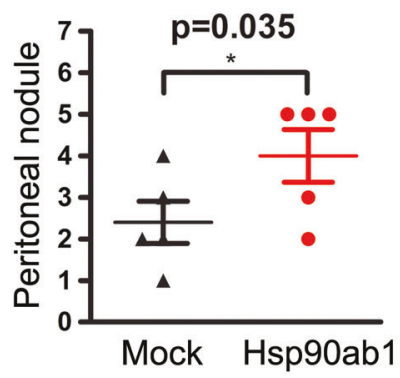

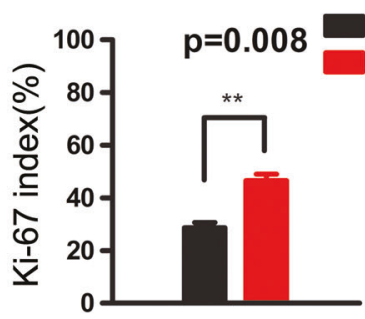

$\mathrm{F}$

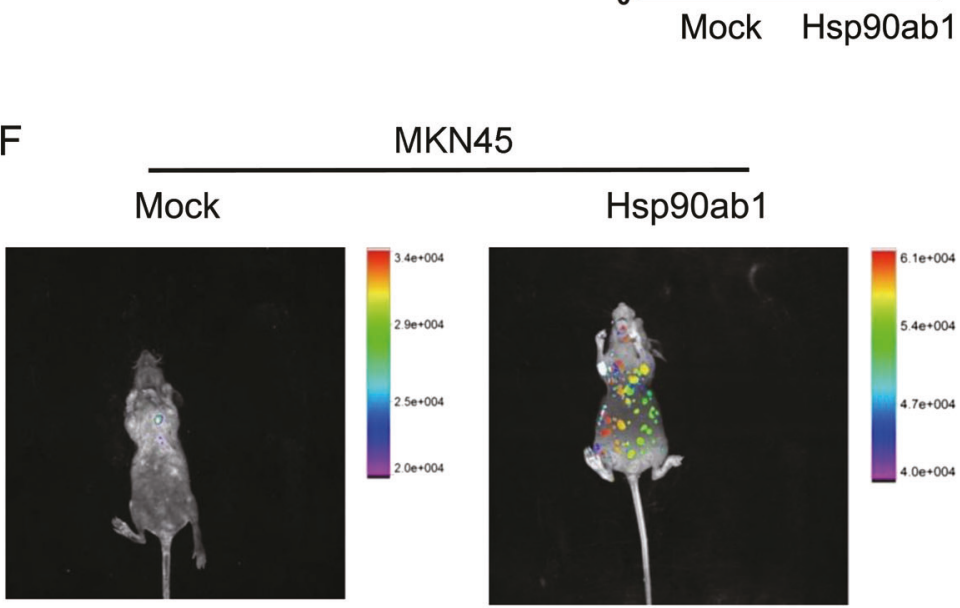

Mock
Hsp90ab
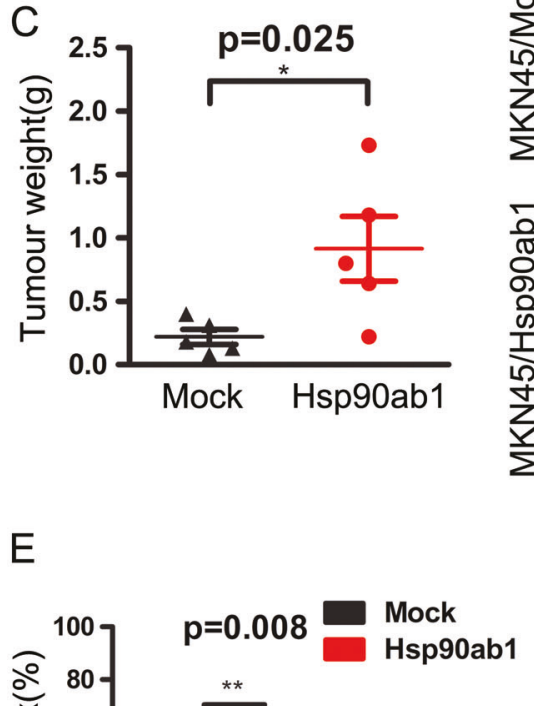

$\mathrm{H}$
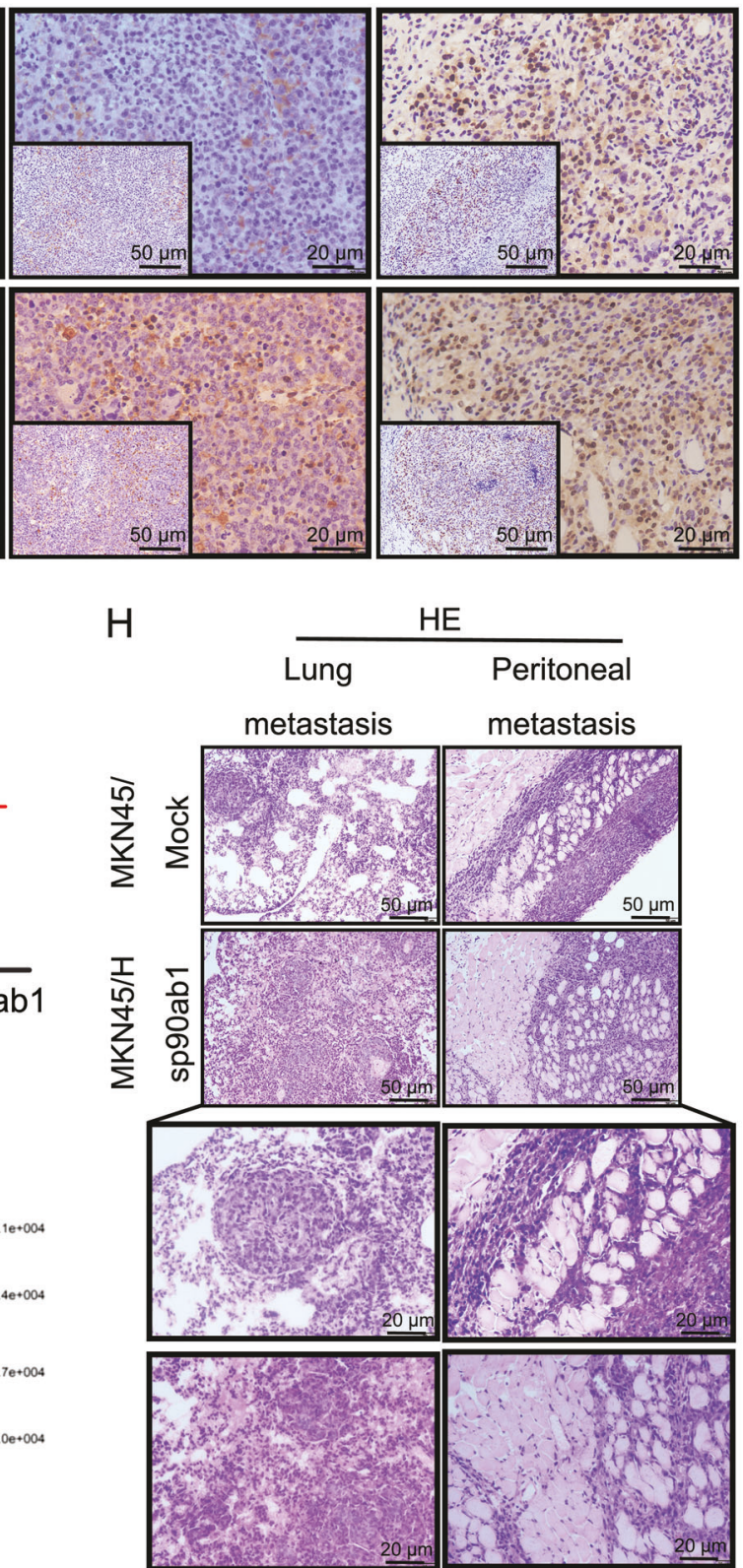

in vitro, significantly higher expression of Ki-67 was detected in Hsp90ab1 overexpressing tumors than that in the control tumors (Fig. 5d, e, $P<0.01$ ).
To determine if Hsp90ab1 play a role in GC metastasis in vivo, we next generated metastatic mouse models. MKN45 cells with and without stable overexpression of 
Fig. 5 The Hsp90ab1 overexpression promoted the growth and metastasis of GC in vivo. a-c Images of subcutaneous tumors of mice injected with Hsp90ab1-overexpression and control stable transfected cells. a MKN45 cells with upregulated Hsp90ab1 expression exhibited enhanced tumor growth in nude mice. b The effect of Hsp90ab1 on GC tumor growth was evaluated based on tumor volume injected with indicated cells. The data of subcutaneous tumors were expressed as mean \pm SD. c Scatter plots of tumor weight derived from indicated cells after subcutaneous implantation. d, e The xenograft tumors was stained with H\&E and the expression of Hsp90ab1 and Ki-67 in the tumors was measured by IHC. Proliferative ability was measured by the Ki-67 index (\%). f-h Hsp90ab1 upregulated cells or control cells were injected into nude mice through the tail vein to evaluate the metastatic potential of cells. The infection efficacy of lentivirus with GFP in vivo (f) and number of metastatic peritoneal nodules (g) in individual mice was analyzed. $\mathbf{h}$ The lung metastasis and metastatic peritoneal nodules sections were stained with $(\mathrm{H} \& \mathrm{E})$. Bottom pictures were local magnification of top pictures. $* P<0.05$; $* * P<0.01$; $* * * P$ $<0.001$

Hsp90ab1 were injected into the tail vein of nude mice. The infection efficacy of the lentivirus in vivo was confirmed by imaging mice on the animal in vivo imaging instrument (Fig. 5f). Mice injected with Hsp90ab1 expressing cells developed more lung metastases and peritoneal metastases compared to those with the control cells (Fig. $5 \mathrm{~g}$, h). These results corroborated previous data and suggested that Hsp90ab1 promoted tumor growth and metastasis in GC cells.

\section{Hsp90ab1 directly interacts with LRP5 and positively regulates LRP5 expression in GC cells}

In order to gain a better understand of the molecular mechanism of Hsp90ab1 in GC cells, we first sought to identify the proteins that directly interacted with Hsp90ab1 in BGC823 cells by immunoprecipitation (IP). The most abundant protein isolated was low-density lipoprotein receptor-related protein 5 (LRP5), as identified by mass spectrometry (Fig. 6a, Table S4). To confirm the proteinprotein interaction between Hsp90ab1 and LRP5, we conducted a Co-IP analysis with an antibody against Hsp90ab1. After immunoprecipitation with Hsp90ab1 conjugated beads, LRP5 was detected in the lysates from both BGC823 and MKN45 cells. In a reciprocal Co-IP with LRP5 conjugated beads, Hsp90ab1 precipitated with LRP5, indicating that endogenous human Hsp90ab1 was physically associated with LRP5 (Fig. 6b). Immunofluorescent staining revealed that Hsp90ab1 and LRP5 co-localized in both cell lines at the cell membrane and in the cytosol (Fig. 6c, Figure S4). Schematic of Hsp90ab1 and LRP5 show the domain of Hsp90ab1 and LRP5 (Fig. 6d, e). Then we performed GST pulldown assays according to this schematic and found that the MD domain of Hsp90ab1 interacted with the domain (1409-1615a) of LRP5 (Figs. 6fh). Finally, we also generated MD domain deletion constructs and found that the deletion of MD fragment within Hsp90ab1 abolished the ability of Hsp90ab1 to interact with LRP5 by GST pulldown assays and exhibited a weak effect on promoting tumor aggressiveness compared with the overexpression group in vitro experiments (Fig. 6i, Figure S5).

When we analyzed the protein sequence of LRP5 in the Compendium of Protein Lysine Modifications database, some ubiquitination sites were predicted within LRP5 (Figure S6). To determine if the protein is ubiquitinated and degraded, we inhibited the synthesis of LRP5 and sitemutated proteins with cycloheximide in HE293 cells. Overexpression of the K143R, K592R and K1561R mutants were more stable than the K802R and K1231R mutants, compared with LRP5-wt, indicating that the Lysto-Arg mutations at these sites abolish LRP5 degradation more or less (Fig. 7a). Then we examined whether MG132, a proteasome inhibitor, could reverse the effect, results of western blot showed that the protein content of LRP5-wt, K802R and K1231R increase obviously (Fig. 7b). Therefore, we believed that K143R, K592R and K1561R were potential Ub acceptor sites which mediated LRP5 ubiquitination by the proteasomal pathway. Because Hsp90ab1 and LRP5 interacted with each other in the domain (14091615a) of LRP5, we focused on the effect of K1561R site mutation on LRP5. Results of western blot showed that both MG132 and Hsp90ab1 did not increase the LRP5 protein level in HEK293 cells compared with the control group (Fig. 7c).

In order to better understand the relationship between Hsp90ab1 and LRP5 in GC cells, we measured LRP5 protein levels in cell lines after lentiviral modulation of Hsp90ab1 expression. In BGC823 cell, transfected with shRNA to knock down Hsp90ab1, LRP5 protein levels decreased as compared to controls (Fig. 7d). We hypothesized that the interaction of Hsp90ab1 and LRP5 sequesters LRP5, which prevents its ubiquitination and proteasomemediated degradation. To confirm that LRP5 expression is affected by ubiquitination pathway mediated by Hsp90ab1, we utilized an in vitro ubiquitination assay to visualize the level of LRP5 ubiquitination. Lentivirus-mediated downregulation of Hsp90ab1 resulted in decrease in LRP5 protein levels. To confirm this is due to protection from inhibiting the ubiquitin mediated degradation, cells treated with $30 \mu \mathrm{M}$ MG132, resulted in accumulation of LRP5 (Fig. 7d). These above data suggested that stabilization of LRP5 was partly mediated by the Hsp90ab1-LRP5 interaction, which inhibited the ubiquitin-mediated degradation of LRP5. 
A
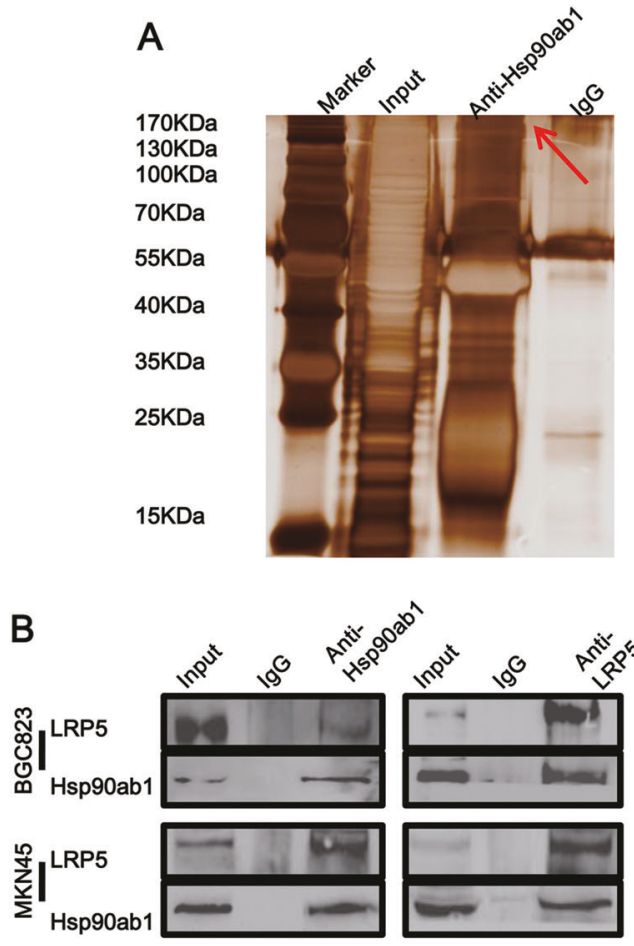

D

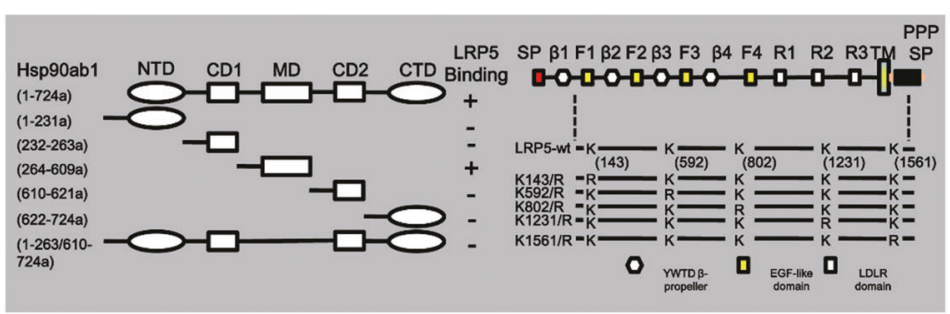

C MKN45
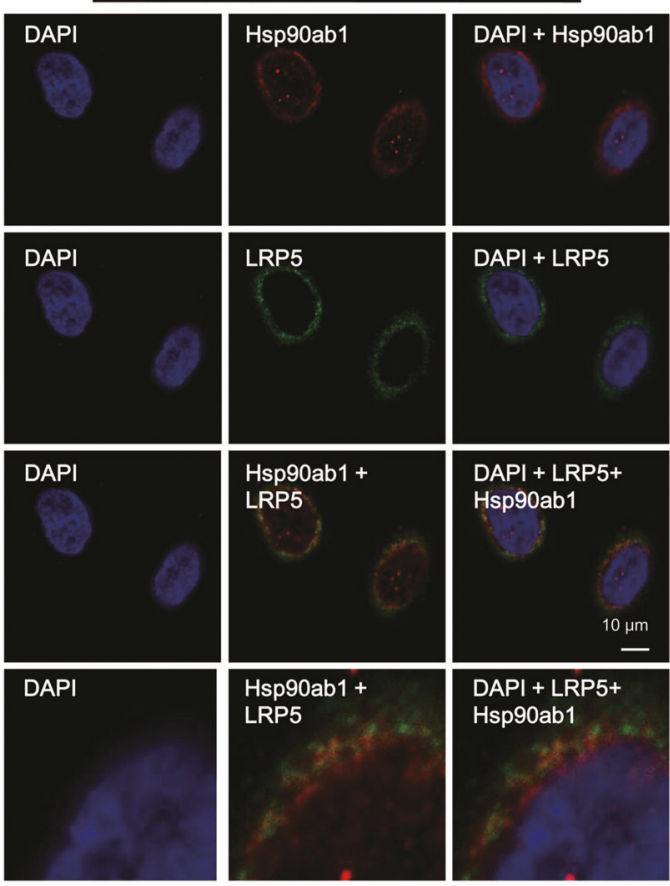

DAPI + LRP5

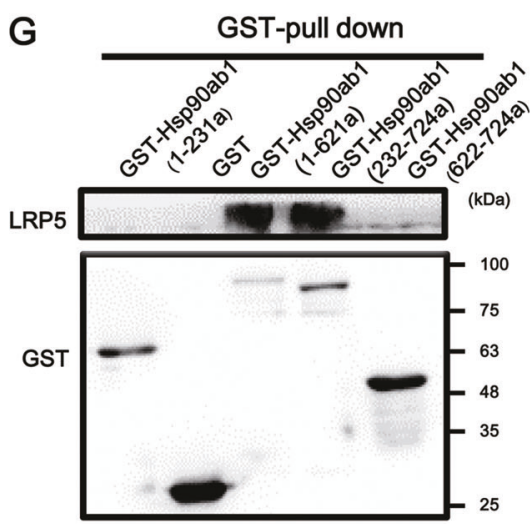

F

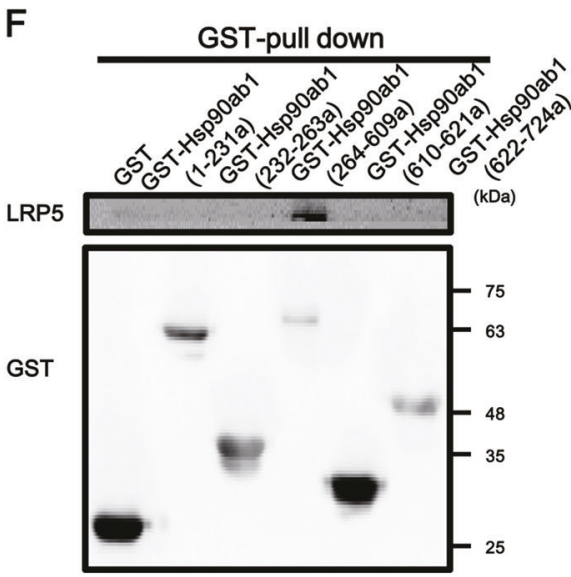

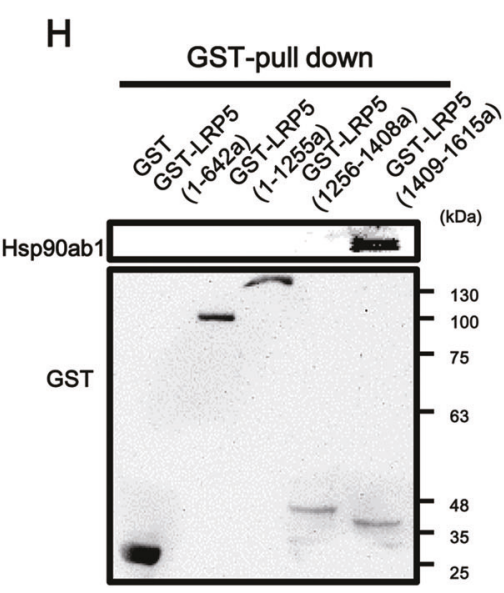

I

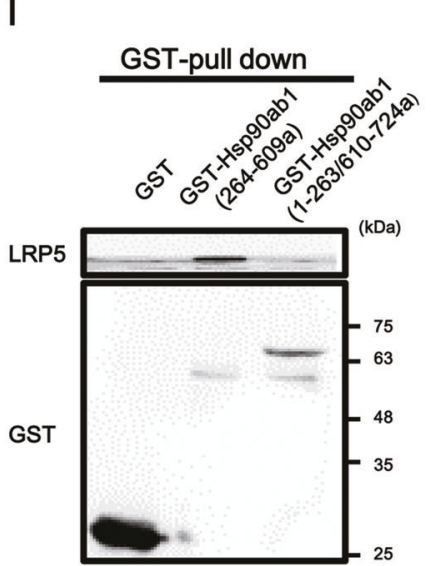


Fig. 6 Hsp90ab1 interacted with LRP5 protein in GC. a Immunoprecipitation assays were used to identify proteins associated with Hsp90ab1. The anti-Hsp90ab1 and IgG antibody were incubated with cell extracts, the bands specific were excised and submitted for mass spectrometry, and LRP5 was identified to be a Hsp90ab1-binding protein. b Co-IP of Hsp90ab1 and LRP5 with each other from proteins of BGC823 and MKN45 cells in GC cells. c Confocal immunofluorescence analysis showed the presence and localization of Hsp90ab1 and LRP5 in the cytoplasm of MKN45 cells. d, e Schematics outlines of both Hsp90ab1 and LRP5 structure features. $\mathbf{f}-\mathbf{h}$ GST pull-down assay results indicated the direct interaction between Hsp90ab1 and LRP5. i Pull-down of LRP5 by GST-tagged Hsp90ab1 fragment (264-609a) and Hsp90ab1 fragment (1-263/610-724a)

\section{Hsp90ab1-mediated EMT in GC cell is mediated by LRP5-induced activation of the AKT and Wnt/B- catenin signaling pathways}

We next sought to understand how Hsp90ab1-mediated protection of LRP5 affected GC cell behavior. LRP5 is a known activator of $\mathrm{Wnt} / \beta$-catenin signaling pathway, which promotes cell growth and migration [33]. Therefore, we hypothesized that overexpression of Hsp90ab1 would increase the levels of $\beta$-catenin and phosphorylated AKT, while knocking down Hsp90ab1 would decrease $\beta$-catenin and phosphorylated AKT. After Hsp90ab1 overexpression, both the levels of phosphorylated AKT and $\beta$-catenin were obviously increased, indicating increased pathway activation. Alternately, knockdown of Hsp90ab1 showed the opposite results. It has been widely known that $\mathrm{Wnt} / \beta$ catenin activation induces the EMT. Therefore, we hypothesized that the expression of Hsp90ab1 would also change the levels of classical EMT markers, such as E-cadherin, $\mathrm{N}$ cadherin, Vimentin, TCF8, Snail, and Slug. We measured levels of these proteins by western blotting in MKN45 and MGC803 cells with up-regulated Hsp90ab1 expression. These cells had increased abundance of $\mathrm{N}$-cadherin, Vimentin, TCF8, Snail, and Slug, but reduced protein abundance of E-cadherin. Hsp90ab1-depleted cells displayed the opposite results (Fig. 7e, Figure S7A). Similarly, knockdown of Hsp90ab1 showed the reduction of LRP5 and $\beta$-catenin, as revealed by immunofluorescence, while overexpression of Hsp90ab1 reverse the effect (Figure S7A, B, Table S5).

To confirm the role of the Hsp90ab1-LRP5 axis in Hsp90ab1-mediated EMT, cell invasion, and metastasis, we hypothesized that LRP5 overexpression and knockdown would reverse the effects of Hsp90ab1 knockdown and overexpression in GC cells. When we induced LRP5 knockdown in Hsp90ab1 overexpressing cells, western blotting indicated reversed EMT markers occurred in these cells (Fig. 7f). To confirm this had a functional consequence, we also measured the migratory capacity of these cells. In alignment with EMT marker reversal, cell migration was also decreased. Alternately, LRP5 overexpression in Hsp90ab1-depleted cells reversed downregulation of EMT markers and impaired migratory activity of GC cells (Fig. 8a, b, Figure S8A, D).

Finally, we evaluated the expression of Hsp90ab1, LRP5, $\beta$-catenin and E-cadherin in tissue samples from GC patients. The expressions of Hsp90ab1, LRP5, $\beta$-catenin and E-cadherin were detected in 36 patients by immunohistochemistry (Fig. 8c, d). Hsp90ab1 levels in these samples were positively correlated with the levels of LRP5 and $\beta$-catenin (LRP5: $r^{2}=0.551, P=0.002$ and $\beta$-catenin: $r^{2}=$ $0.351, P=0.036$ ). However, there was no significant correlation between Hsp90ab1 expression and E-cadherin $\left(\mathrm{r}^{2}\right.$ $=0.039, P=0.820$ ). Taken together, these data supported the hypotheses that Hsp90ab1 promoted the invasion and migration of GC cells via the formation and activation of Hsp90ab1-LRP5 axis.

\section{Discussion}

In the present study, we demonstrated that Hsp90ab1 was upregulated in GC cells, which resulted in significantly worse patient outcomes and higher cancer metastasis rate. We showed that this was a result of Hsp90ab1 mediated stabilization of LRP5, which activated both AKT and Wnt/ $\beta$-catenin signaling pathways. It is well known that Hsp90ab1 is a molecular chaperone that is required for the formation, stability and function of its client proteins, many of which promote cancer cell growth and survival [23]. Growing evidence has revealed that Hsp90ab1 overexpression occurs in various different cancers, and this overexpression plays an integral role in the multistep processes leading to carcinogenesis and invasion [24, 30, 34, 35]. However, the significance and underlying mechanism of Hsp90ab1 in GC was previously unknown.

We first confirmed that Hsp90ab1 was remarkably upregulated in GC tissues and it was significantly associated with aggressive stages and unfavorable prognosis of GC patients. By Kaplan-Meier survival curve analysis, we found that the disease-free survival of patients with Hsp90ab1 high expression was significantly shorter than that of other patients. In particular, the expression of Hsp90ab1 was significantly associated with shorter survival for patients with stage III/IV GC. Furthermore, we found that Hsp90ab1 was an independent prognostic factor for overall survival of GC patients, indicated by multivariate cox regression analysis. Besides, Hsp90ab1 could increase the tolerance of GC cells to chemotherapeutic agents, such as HIF1 $\alpha, \mathrm{Cdc} 37$, to affect the prognosis of patients[36, 37]. These data suggested that Hsp90ab1 might be a valuable new prognostic marker for the screening, diagnosis, and prognosis for GC patients. 
A

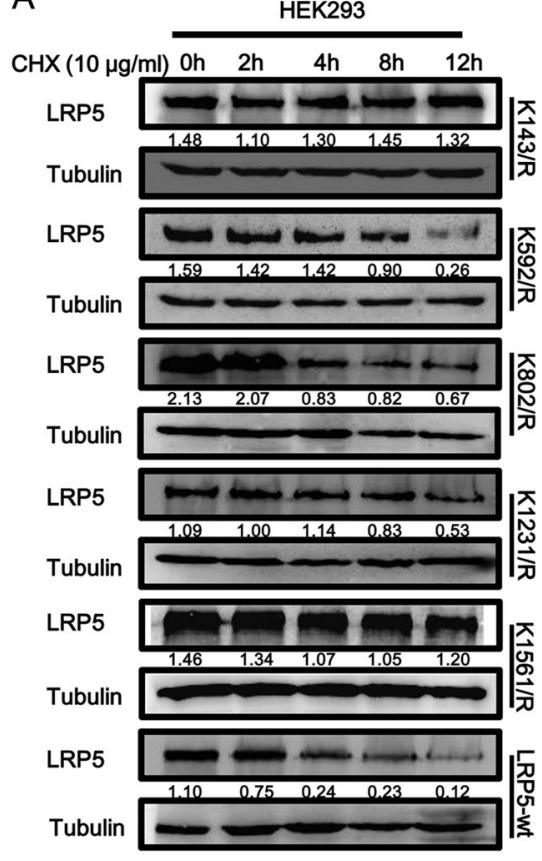

B

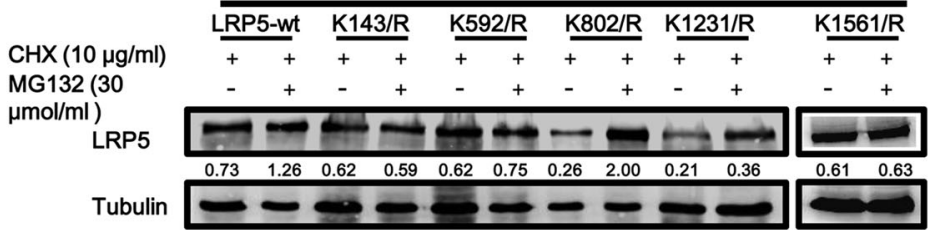

C

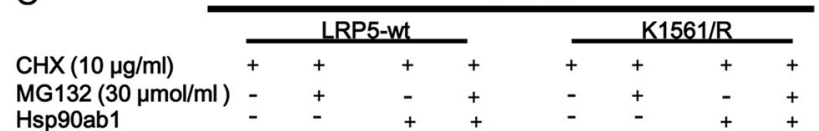

LRP5

Hsp90ab1

Tubulin

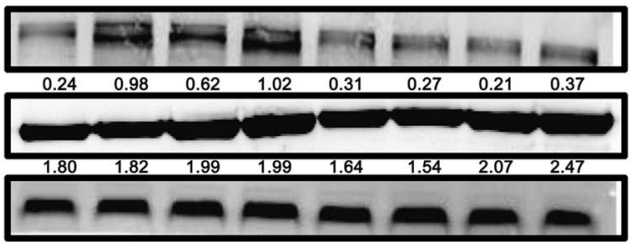

D

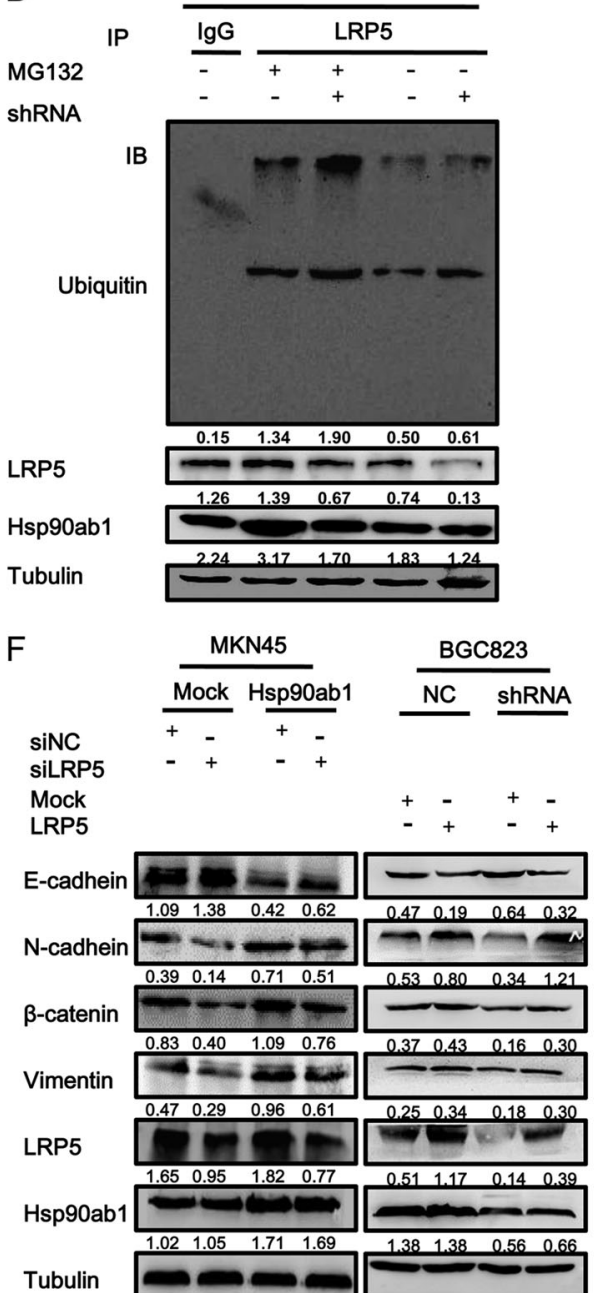

Previously, Hsp90ab1 has been implicated to have an oncogenic role in laryngeal carcinoma [38], non-small-cell lung cancer [30, 39], and breast cancer [40, 41] by promoting tumorigenesis. In this study, we demonstrated that Hsp90ab1 was both necessary and sufficient to promote the invasion and migration of GC tumorigenesis in vitro and 
Fig. 7 Hsp90ab1 inhibited the ubiquitin degradation of LRP5 and promoted the proliferation and invasion of GC cells by activating AKT and Wnt/ $\beta$-catenin signaling pathway. a HEK $293 \mathrm{~T}$ cells were transiently transfected with LRP5-wt plasmid or the K142R, K592R, K802R, K1231R, and K1561R mutants, and treated with CHX at 10 $\mu \mathrm{g} / \mathrm{ml}$ for the indicated times. Cell lysates were assessed by Western blot analysis. b HEK $293 \mathrm{~T}$ cells were transiently transfected with LRP5-wt plasmid or the K142R, K592R, K802R, K1231R and K1561R mutants, and treated with CHX at $10 \mu \mathrm{g} / \mathrm{ml}$ and MG132 at 30 $\mu \mathrm{M}$ for $12 \mathrm{~h}$. Cell lysates were assessed by Western blot analysis. c HEK 293 T cells were transiently co-transfected with LRP5-wt plasmid or the K1561R mutants and Hsp90ab1, and treated with CHX at $10 \mu \mathrm{g} / \mathrm{ml}$ and MG132 at $30 \mu \mathrm{M}$ for $12 \mathrm{~h}$. Cell lysates were assessed by Western blot analysis. d Hsp90ab1 increased the LRP5 level by inhibiting its ubiquitin-mediated degradation in BGC823 cells. e Western blotting analyses of the levels of $\mathrm{Akt}, \mathrm{Wnt} / \beta$-catenin signaling pathways and classical EMT markers in GC cells treated with Hsp90ab1 or shRNA1. f Western blotting analyses of the levels of Hsp90ab1, LRP5 and classical EMT markers in GC cells treated with co-transfection of Hsp90ab1 and siLRP5 or shHsp90ab1 and LRP5

in vivo, thereby indicating an invasion-promoting effect in GC progression. In combination with prior findings, our data supported the hypothesis that Hsp90ab1 plays an important role in tumorigenesis in multiple tumor types.

Recently, it was proposed that Hsp90ab1 might stabilize Cdc25A, increasing its expression levels and promoting cell-cycle activation in pancreatic carcinoma cells [42]. Likewise, Hsp90ab1 has also been shown to promote leukemia cell proliferation by preventing the autoubiquitination and degradation of c-IAP1, a member of the inhibitor of apoptosis protein (IAP) family [43]. Beside, Hsp90 has been reported to interact with AKT to induce autophagy through activation of the AKT/mTOR pathway in lung cancer [44]. However, we suspect that apoptosis inhibition is not the only function of Hsp90ab1, and that its role in GC may be mediated by its role as a molecular chaperone.

In our study, we showed that LRP5 was a novel binding partner of Hsp90ab1 in GC cells. LRP5 has been reported to be an indispensable coreceptor that regulates the activity and function of $\mathrm{Wnt} / \beta$-catenin signaling pathway [33]. LRP5 binds to Frizzled $(\mathrm{Fz})$ and Dishevelled Segment Polarity Protein 2 (Dvl-2v) for the initiation of Wnt signaling [45, 46], which results in the translocation of the transcription co-activator protein, $\beta$-catenin, into the nucleus. Additionally LRP5 overexpression has previously been shown to increase $\beta$-catenin expression and activation in malignant cancers [47-49]. In the present study, we revealed that Hsp90ab1 reduced the ubiquitin-mediated proteasome degradation of LRP5, resulting in LRP5 upregulation in GC cells. We confirmed the role of LRP5 in GC by showing that knockdown of LRP5 partly counteracted the malignant phenotypes mediated by Hsp90ab1. However, the exact mechanisms dictating LRP5 ubiquitination and activation within GC cells require further exploration. Although further studies need to be carried out, these results collectively demonstrated that the role of LRP5 is essential in Hsp90ab1-mediated cancer metastasis.

The EMT occurs via activation of both AKT and and $\mathrm{Wnt} / \beta$-catenin signaling pathways, which may contribute to tumor metastasis [49-52]. Our results illustrated that Hsp90ab1 was a new interacting partner of LRP5, an indispensable coreceptor of the Wnt signaling pathway. Enhanced canonical Wnt signaling increases the stabilization of $\beta$-catenin and induces the nuclear import of $\beta$-catenin, which increases the levels of transcription factors TCF, Snail, and Slug. These proteins regulate the activation of the EMT. Additionally, the EMT has previously been shown to be a positive regulator of growth, migration, and invasion in GC [53-55]. As confirmed by the current research, Hsp90ab1 can increase the levels of phosphorylated AKT and $\beta$-catenin to promote the invasion and migration of GC. These data collectively suggested that Hsp90ab1 inactivated AKT and $\mathrm{Wnt} / \beta$-catenin signaling pathways.

In conclusion, this study investigated the potential role of Hsp90ab1 in GC cell invasion and metastasis. Our data suggested that Hsp90ab1 could bind to LRP5 and inhibit the ubiquitin degradation of LRP5 and subsequently activated the AKT and Wnt/ $\beta$-catenin signaling pathways (Fig. 9). Hsp90ab1 may contribute significantly to develop specific drugs targets for GC patients.

\section{Materials and methods}

\section{Tumor tissue sample}

Two independent cohorts involving 332 people were involved in the study. In cohort 1, 150 primary GC patients who underwent radical surgery at Nanfang Hospital between 2013 and 2016 were enrolled in the study. All patients enrolled provided written informed consent prior to obtaining the study specimens of patients in accordance with the ethical protocols of the Ethics Committee of Nanfang Hospital, Southern Medical University. No patients received preoperative chemotherapy or radiotherapy before surgery. Among these tissue biopsies, 102 cases of the fresh-frozen tumor samples and matched adjacent non-tumor tissues were used for quantitative realtime (qRT)-PCR and 12 cases for western blotting analyses. Other 36 cases were used to investigate the correlativity between Hsp90ab1 and the biomarkers of EMT. For cohort 2, 182 cases of samples were enrolled for immunochemical stain for Hsp90ab1 using a gastric adenocarcinoma tissue microarrays (HStm-Ade180Sur-06 and HStmAde167Sur01; Shanghai Outdo Biotech), which was obtained from the National Engineering Center For Biochip. The 

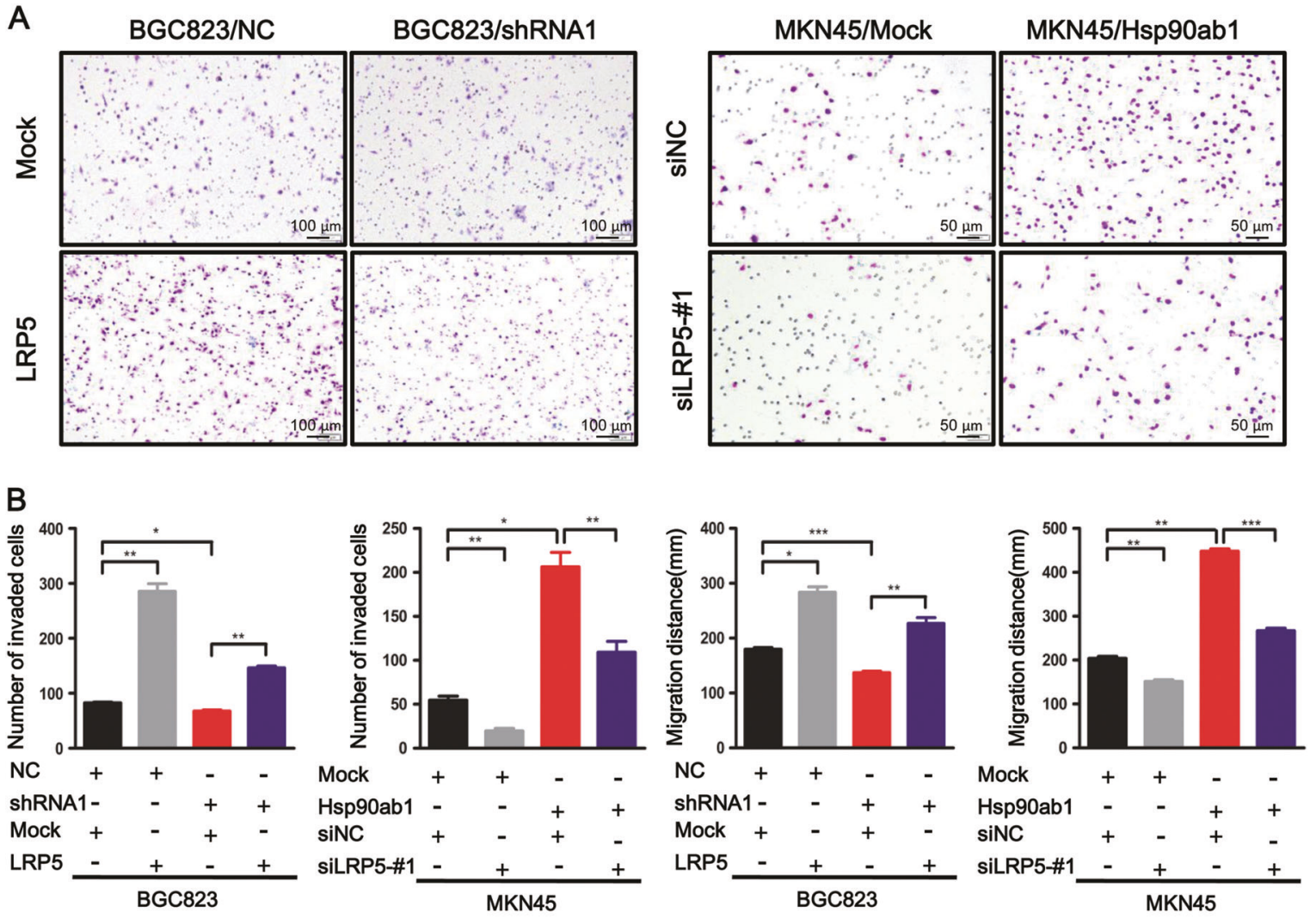

C
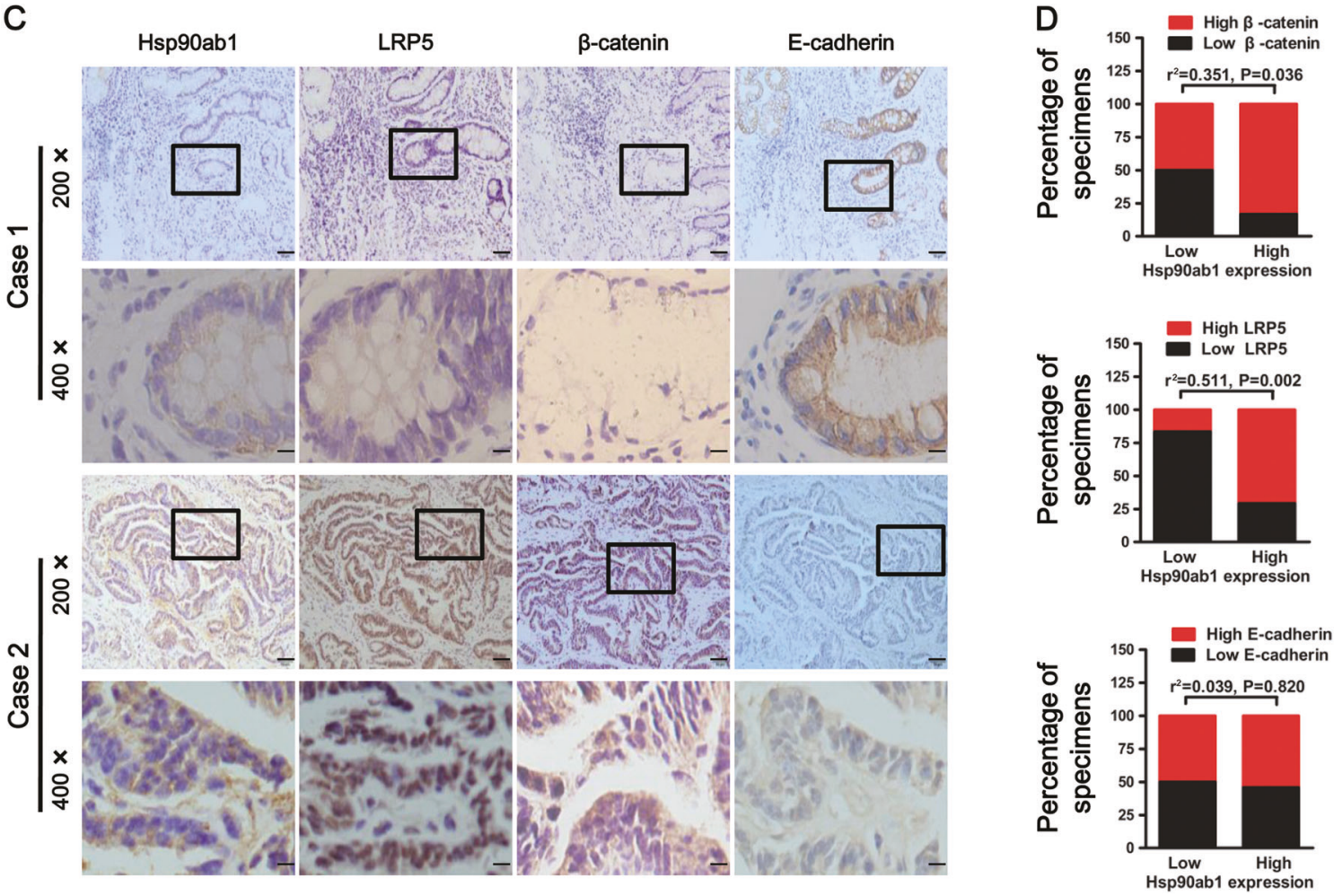

clinicopathologic features of patients, including the age, gender, tumor location, size of primary tumor, tumor differentiation and tumor node metastasis classification, were recorded and archived in the National Engineering Center For Biochip. 
Fig. 8 LRP5 was positively correlated with Hsp90ab1 in GC cells and tissues. a Effects of co-transfection of shRNA1 and LRP5 or Hsp90ab1 and siLRP5-\#1 on cell invasion and migration by Matrigel invasion assays and Scratch-wound-healing assays. b Graphical illustration of statistical result of co-transfection of shRNA2 and LRP5 or Hsp90ab1 and siLRP5-\#1 on cell invasion and migration. $\mathbf{c}$ The Hsp90ab1 expression was positively correlated with LRP5 and $\beta$ catenin, but it was meaningless correlation with E-cadherin in $36 \mathrm{GC}$ specimens. Representative figures of two cases were shown. Bottom pictures were local magnification of top pictures. d Graphical illustration of the levels of Hsp90ab1 expression that were associated with the levels of $\beta$-catenin, LRP5, and E-cadherin in $36 \mathrm{GC}$ specimens the Department of Pathology of Nanfang Hospital, Southern Medical University.

\section{RNA extraction and qRT-PCR}

RNA extraction and qRT-PCR were performed as previously described [56], with the following modifications. The primers were listed in Table S1. GAPDH was chosen as an internal quantitative reference. Each experiment was conducted in triplicate.

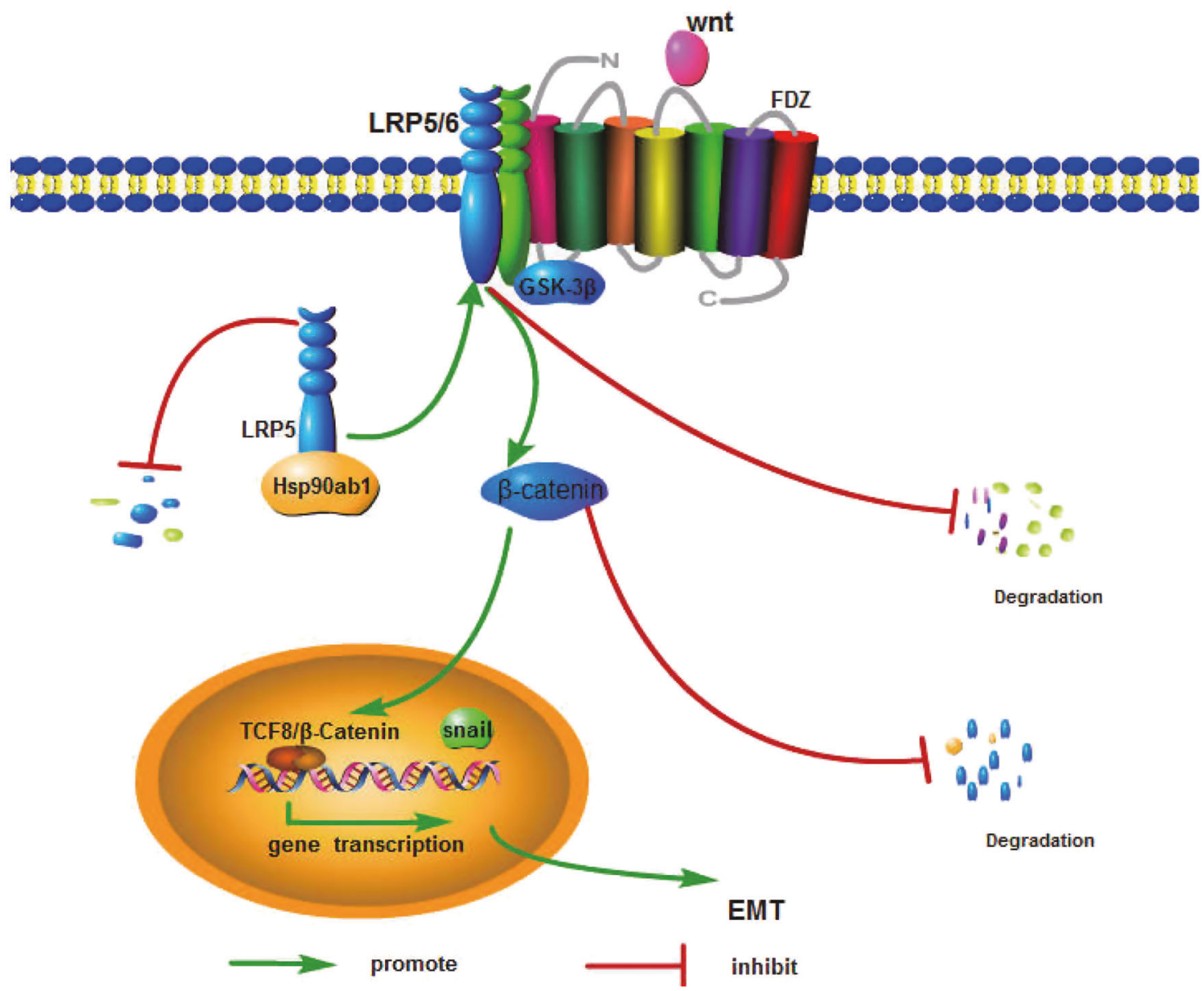

Fig. 9 Schematic illustration of the molecular mechanism of Hsp90ab1 promoting the development of gastric cancer by a hypothetical model

\section{Cell line preparation}

Human GC cell lines, including AGS, MKN45, MKN28, MGC803, NCI-N87 and SGC7901, were obtained from the Committee of Type Culture Collection of Chinese Academy of Sciences (Shanghai, China). Additionally, three human GC cell lines (BGC823, BGC803, MGC823), the gastric mucosal cell GSE-1 and HEK293 were kindly provided by

\section{Western blot analysis}

The protein expression of cell lines as well as the freshfrozen tumor samples and matched adjacent non-tumor tissue was performed in radio immunoprecipitation assay buffer (KeyGEN, Nanjing, China). $50 \mu \mathrm{g}$ of protein lysate was loaded on a $10 \%$ SDS-PAGE gel and separated by 
electrophoresis. The proteins were transferred to polyvinylidene fluoride membranes (Millipore, Billerica, MA, USA). The membranes were incubated with various antibodies (Supplementary Table S2) overnight at $4{ }^{\circ} \mathrm{C}$. The primary antibodies were detected using corresponding secondary antibodies (Supplementary Table S2). All experiments were repeated in triplicate, and the representative results were shown.

\section{Immunohistochemistry analysis}

Immunohistochemistry (IHC) was performed as previously described [57], with the following modifications. The slides were incubated overnight with primary antibodies against Hsp90ab1 (1:500, ab203085, Abcam), E-cadherin (1:100, ZM-0092, Maixin Biotech), $\beta$-catenin (1:100, \#8480, CST) and LRP5 (1:100, D260566, Sangon Biotech), Ki67 (1:100, $\mathrm{ZM}-0502$, Maixin Biotech) at $4{ }^{\circ} \mathrm{C}$. The images were analyzed as described in Supplementary Methods.

\section{Construction of plasmid and transfection}

Expression of human Hsp90ab1 was knocked down using a lentivirus vector carrying a specific Hsp90ab1 short-hairpin RNA (shRNA) (Vigenebio, Maryland, USA) or siRNA (GenePharma, Shanghai, China), and LRP5 was knocked down using siRNA (GenePharma, Shanghai, China) as previously described [57, 58]. The target sequences were listed in Table S1 for details. Expression of human Hsp90ab1 was upregulated using a lentivirus vector (Vigenebio, Maryland, USA) and LRP5 was up-regulated using a plasmid (Sino Biological Inc., Beijing, China) as previously described [57, 58]. Flag-LRP5 lysines (K) 143, K592, K806, K1231 and K1561 separately mutated to arginine (R), and plasmids termed "LRP5 K143R", "LRP5 K592R", "LRP5 K802R", "LRP5 K1231R" and "LRP5 K1561R" mutants were generated by Mut Express II Fast Mutagenesis Kit V2 (Vazyme Biotech Co.,Ltd, Nanjing, China) and confirmed by sequencing. Plasmids and siRNAs were transient transfected into cells using Lipofectamine 2000, according to the manufacturer's instructions (Thermo Fisher Scientific, USA).

\section{Cell proliferation, cell cycle, colony formation, wound healing, matrigel invasion assays}

The Cell counting kit-8 (CCK-8), cell cycle, colony formation, cell migration and invasion assays were performed as described previously $[59,60]$.

\section{EdU incorporation assay}

GC cell proliferation was determined in vitro using the CellLight TM EdU staining kit (RiboBio, Guangzhou, China) according to manufacturer instructions. The proportion of positive cells in each well were counted.

\section{Oxaliplatin-sensitivity assay}

The oxaliplatin-sensitivity of cells was measured as described previously [61].

\section{Xenograft tumor model}

All animal experiments procedures were approved and conducted by the Southern Medical University Animal Care and Use Committee and the experiments were in accordance with the guidelines for the ethical treatment of animals. To determine the function of the Hsp90ab1 in tumor formation in vivo, cells stably expressing Hsp90ab1 were injected subcutaneously into nude mice. Tumor size was measured and calculated as previously described [57]. To explore the effects of Hsp90ab1 on tumor metastasis in vivo, cells stably expressing the indicated Hsp90ab1 or constructs were injected through the lateral tail vein of nude mice. 1 month later, the mice were sacrificed by cervical vertebra dislocation. Liver, lung and peritoneum were harvested, and prepared for hematoxylin and eosin and IHC staining as described above.

\section{Co-immunoprecipitation}

Co-immunoprecipitation of proteins was performed as previously described [62], with the following modifications. Supernatants were incubated with protein $A / G$ beads (Selleck Chemicals, Houston, USA) overnight at $4{ }^{\circ} \mathrm{C}$ to pre-clear the lysates. The pre-cleared lysates were incubated at $4{ }^{\circ} \mathrm{C}$ with primary antibody and protein $\mathrm{A} / \mathrm{G}$ plus overnight according to the manufacturer's instructions. The protein A/G-antibody-antigen complex was concentrated by centrifugation at $1000 \times g$ for $10 \mathrm{~min}$ at $4{ }^{\circ} \mathrm{C}$ and washed with PBS; the procedure was repeated three times. Immunoprecipitated proteins were then separated by SDS-PAGE, and visualized by Western blot and silver staining (Byeotime, Shanghai, China). The gels were digested for LCMS/MS analysis as previously described [63], The detailed process was listed in the supplementary method.

\section{Immunofluorescence analysis}

Cells were cultured on glass coverslips for $12 \mathrm{~h}$ and fixed with ethanol for $30 \mathrm{~min}$ at $-20^{\circ} \mathrm{C}$. After fixation, cells were permeabilized with $0.25 \%$ Triton $\mathrm{X}-100$ for $10 \mathrm{~min}$ at room temperature and blocked in $10 \%$ normal blocking serum at room temperature for $10 \mathrm{~min}$, then incubated overnight at 4 ${ }^{\circ} \mathrm{C}$ with primary antibodies against Hsp90ab1 (1:200, ab203085, Abcam) and LRP5 (1:100, sc390267, Santa 
Cruz), and $\beta$-catenin (1:200, \#8480, CST). The next day, slides were incubated with Alexa Fluor 488 and Alexa Fluor 594 labeled secondary antibodies (1:1000, Proteintech Group Inc, Wuhan, China) for $1 \mathrm{~h}$ at room temperature. To visualize nuclei, slides were incubated with 6-diamidino-2phenylindole (DAPI; KeyGEN, Nanjing, China).

\section{Glutathione S-transferase protein pull-down assay}

GST-pET-41a( + )-Hsp90ab1 (1-231a), (232-263a), (264609a), (610-621a), (622-724a), (1-621a), (232-724a), and (1-263/610-724a), GST-pET-41a(+)-LRP5 (1-642a), (11255a), (1256-1408a) and (1409-1615a) (Vigenebio, Maryland, USA) were transformed into Escherichia coli strain BL21 (DE3) and induced for the expression of GST or GST-fusion protein by IPTG. And these proteins were purified using the Beaver beads TM GSH kit (Beaver Biosciences Inc, Suzhou China) according to the manufacturer's instructions. Pull-down assays were performed by incubating GST fusion protein with the cell lysates of HEK293T cells which were transfected with Flag-LRP5 at $4{ }^{\circ} \mathrm{C}$ for $4 \mathrm{~h}$. Then the bead-bound protein complexes were then washed and detected by western blot.

\section{Protein ubiquitination assay}

Hsp90ab1 expression lentiviral vector or empty lentiviral vector were transfected into BGC823 cells. Cells were incubated in the presence or absence of $30 \mu \mathrm{M}$ MG132 (Selleck, Houston, USA) for $24 \mathrm{~h}$ and lysed in RIPA buffer supplemented with proteinase inhibitor. Then immunoprecipitation was carried out with anti- Hsp90ab1 or anti-IgG antibodies following the protocol above. The immunoprecipitated proteins were subjected to western blot using antiubiquitin (Proteintech Group Inc., Wuhan, China) to evaluate the ubiquitination level.

\section{Statistical analysis}

All statistical analyses were performed using SPSS version 19.0 software (SPSS, Chicago, IL, USA). The correlation between the protein expression and clinicopathological factors in GC tumor tissue and the paired normal tissue was determined by Pearson's chi-square test. Survival curves were plotted according to the Kaplan-Meier method and were compared with the log-rank test. The volumes of xenograft tumor in nude mice from the experimental and control groups were compared with Student's t-test. The statistical analysis of cell proliferation curve, and migration rate between different cell groups was carried out by Oneway ANOVA and the $\chi^{2}$ test. Values of $P<0.05$ were considered statistically significant.
Acknowledgements This work was supported by the grants from the State's Key Project of Research and Development Plan (2017YFC0108300, 2017YFC0108301), National Natural Science Foundation of China (81672446), Guangdong Provincial Science and Technology Key Project (2014A020215014), Research Fund of Public Welfare in the Health Industry, the National Health and Family Planning Commission of China (201402015), the Southern Medical University Clinical Research Start-Up Project (LC2016ZD003), Guangzhou Science and Technology Project (201400000004-5) and the Key Clinical Specialty Discipline Construction Program ([2011] 170).

Author contributions This study was conceived and supervised by Professor GL and SW. Experiments were carried out by HW and GD. Data analysis were conducted by HW and MA. ZX provided technical support for IHC. TM, JY, and HL gave assistance in collecting tissue samples and clinical data. Funding was obtained by GL. The manuscript was written by HW. The manuscript was commented and revised by GL and SW. All authors were involved in writing the paper and had final approval of the submitted and published versions.

\section{Compliance with ethical standards}

Conflict of interest The authors declare that they have no conflict of interest.

Open Access This article is licensed under a Creative Commons Attribution 4.0 International License, which permits use, sharing, adaptation, distribution and reproduction in any medium or format, as long as you give appropriate credit to the original author(s) and the source, provide a link to the Creative Commons license, and indicate if changes were made. The images or other third party material in this article are included in the article's Creative Commons license, unless indicated otherwise in a credit line to the material. If material is not included in the article's Creative Commons license and your intended use is not permitted by statutory regulation or exceeds the permitted use, you will need to obtain permission directly from the copyright holder. To view a copy of this license, visit http://creativecommons. org/licenses/by/4.0/.

\section{References}

1. Pavlakis N, Sjoquist KM, Martin AJ, Tsobanis E, Yip S, Kang $\mathrm{YK}$, et al. Regorafenib for the treatment of advanced gastric cancer (INTEGRATE): a multinational placebo-controlled phase II trial. J Clin Oncol. 2016;34:2728-35.

2. Zheng R, Zeng H, Zhang S, Chen W. Estimates of cancer incidence and mortality in China, 2013. Chin J Cancer. 2017;36:66.

3. Wang L, Yin J, Wang X, Shao M, Duan F, Wu W, et al. C-type lectin-like receptor 2 suppresses AKT signaling and invasive activities of gastric cancer cells by blocking expression of phosphoinositide 3-kinase subunits. Gastroenterology. 2016;150:1183-1195e16.

4. Hu Y, Huang C, Sun Y, Su X, Cao H, Hu J, et al. Morbidity and mortality of laparoscopic versus open D2 distal gastrectomy for advanced gastric cancer: a randomized controlled trial. J Clin Oncol. 2016;34:1350-7.

5. Wang K, Liang Q, Li X, Tsoi H, Zhang J, Wang H, et al. MDGA2 is a novel tumour suppressor cooperating with DMAP1 in gastric cancer and is associated with disease outcome. Gut. 2016;65:1619-31.

6. Okubo K, Uenosono Y, Arigami T, Yanagita S, Matsushita D, Kijima $T$. et al. Clinical significance of altering epithelial- 
mesenchymal transition in metastatic lymph nodes of gastric cancer. Gastric Cancer. 2017;20:802-10.

7. Kurashige J, Mima K, Sawada G, Takahashi Y, Eguchi H, Sugimachi K, et al. Epigenetic modulation and repression of miR$200 \mathrm{~b}$ by cancer-associated fibroblasts contribute to cancer invasion and peritoneal dissemination in gastric cancer. Carcinogenesis. 2015;36:133-41.

8. Liu SH, Lee WJ, Lai DW, Wu SM, Liu CY, Tien HR, et al. Honokiol confers immunogenicity by dictating calreticulin exposure, activating ER stress and inhibiting epithelial-tomesenchymal transition. Mol Oncol. 2015;9:834-49.

9. Wu SM, Lin WY, Shen CC, Pan HC, Keh-Bin W, Chen YC, et al. Melatonin set out to ER stress signaling thwarts epithelial mesenchymal transition and peritoneal dissemination via calpainmediated C/EBPbeta and NFkappaB cleavage. J Pineal Res. 2016;60:142-54.

10. Zhang P, Tang WM, Zhang H, Li YQ, Peng Y, Wang J, et al. MiR-646 inhibited cell proliferation and EMT-induced metastasis by targeting FOXK1 in gastric cancer. $\mathrm{Br} \mathrm{J}$ Cancer. 2017; 117:525-34.

11. Cai J, Feng D, Hu L, Chen H, Yang G, Cai Q, et al. FAT4 functions as a tumour suppressor in gastric cancer by modulating Wnt/beta-catenin signalling. Br J Cancer. 2015;113:1720-9.

12. Kalluri R, Weinberg RA. The basics of epithelial-mesenchymal transition. J Clin Invest. 2009;119:1420-8.

13. Huang J, Xiao D, Li G, Ma J, Chen P, Yuan W, et al. EphA2 promotes epithelial-mesenchymal transition through the Wnt/betacatenin pathway in gastric cancer cells. Oncogene. 2014;33:273747.

14. Qiu S, Xiao Z, Piao C, Zhang J, Dong Y, Cui W, et al. AMPKalpha2 reduces renal epithelial transdifferentiation and inflammation after injury through interaction with CK2beta. J Pathol. 2015;237:330-42.

15. Shi ZM, Wang L, Shen H, Jiang CF, Ge X, Li DM, et al. Downregulation of miR-218 contributes to epithelialmesenchymal transition and tumor metastasis in lung cancer by targeting Slug/ZEB2 signaling. Oncogene. 2017;36:2577-88.

16. Li CF, Huang WW, Wu JM, Yu SC, Hu TH, Uen YH, et al. Heat shock protein 90 overexpression independently predicts inferior disease-free survival with differential expression of the alpha and beta isoforms in gastrointestinal stromal tumors. Clin Cancer Res. 2008;14:7822-31.

17. Jeon YK, Park CH, Kim KY, Li YC, Kim J, Kim YA, et al. The heat-shock protein 90 inhibitor, geldanamycin, induces apoptotic cell death in Epstein-Barr virus-positive NK/T-cell lymphoma by Akt down-regulation. J Pathol. 2007;213:170-9.

18. Whitesell L, Lindquist SL. HSP90 and the chaperoning of cancer. Nat Rev Cancer. 2005;5:761-72.

19. Tsutsumi S, Beebe K, Neckers L. Impact of heat-shock protein 90 on cancer metastasis. Future Oncol. 2009;5:679-88.

20. Ohkubo S, Kodama Y, Muraoka H, Hitotsumachi H, Yoshimura C, Kitade M, et al. TAS-116, a highly selective inhibitor of heat shock protein 90alpha and beta, demonstrates potent antitumor activity and minimal ocular toxicity in preclinical models. Mol Cancer Ther. 2015;14:14-22.

21. Yano M, Naito Z, Yokoyama M, Shiraki Y, Ishiwata T, Inokuchi $\mathrm{M}$, et al. Expression of hsp90 and cyclin D1 in human breast cancer. Cancer Lett. 1999;137:45-51.

22. Hamamoto R, Toyokawa G, Nakakido M, Ueda K, Nakamura Y. SMYD2-dependent HSP90 methylation promotes cancer cell proliferation by regulating the chaperone complex formation. Cancer Lett. 2014;351:126-33.

23. Park S, Park JA, Yoo H, Park HB, Lee Y. Proteasome inhibitorinduced cleavage of HSP90 is mediated by ROS generation and caspase 10-activation in human leukemic cells. Redox Biol. 2017;13:470-6.
24. Meng J, Liu Y, Han J, Tan Q, Chen S, Qiao K, et al. Hsp90beta promoted endothelial cell-dependent tumor angiogenesis in hepatocellular carcinoma. Mol Cancer. 2017;16:72.

25. Perotti C, Liu R, Parusel CT, Bocher N, Schultz J, Bork P, et al. Heat shock protein-90-alpha, a prolactin-STAT5 target gene identified in breast cancer cells, is involved in apoptosis regulation. Breast Cancer Res. 2008;10:R94.

26. Pick E, Kluger Y, Giltnane JM, Moeder C, Camp RL, Rimm DL, et al. High HSP90 expression is associated with decreased survival in breast cancer. Cancer Res. 2007;67:2932-7.

27. Westekemper H, Karimi S, Susskind D, Anastassiou G, Freistuhler M, Steuhl KP, et al. Expression of HSP 90, PTEN, and Bcl2 in conjunctival melanoma. Br J Ophthalmol. 2011;95:853-8.

28. Wu Y, Huang B, Liu Q, Liu Y. Heat shock protein 90-beta overexpression is associated with poor survival in stage I lung adenocarcinoma patients. Int J Clin Exp Pathol. 2015;8:8252-9.

29. Canella A, Welker AM, Yoo JY, Xu J, Abas FS, Kesanakurti D, et al. Efficacy of Onalespib, a long-acting second-generation HSP90 inhibitor, as a single agent and in combination with temozolomide against malignant gliomas. Clin Cancer Res. 2017;23:6215-26.

30. Biaoxue R, Xiling J, Shuanying Y, Wei Z, Xiguang C, Jinsui W, et al. Upregulation of Hsp90-beta and annexin A1 correlates with poor survival and lymphatic metastasis in lung cancer patients. $\mathbf{J}$ Exp Clin Cancer Res. 2012;31:70.

31. Choi HJ, Joo HS, Won HY, Min KW, Kim HY, Son T. et al. Role of RBP2-induced ER and IGF1R-ErbB signaling in tamoxifen resistance in breast cancer. J Natl Cancer Inst.2018;110:400-10.

32. Chen Y, Camacho SC, Silvers TR, Razak AR, Gabrail NY, Gerecitano JF, et al. Inhibition of the nuclear export receptor XPO1 as a therapeutic target for platinum-resistant ovarian cancer. Clin Cancer Res. 2017;23:1552-63.

33. DeBruine ZJ, Ke J, Harikumar KG, Gu X, Borowsky P, Williams BO, et al. Wnt5a promotes Frizzled-4 signalosome assembly by stabilizing cysteine-rich domain dimerization. Genes Dev. 2017;31:916-26.

34. Cheng Q, Chang JT, Geradts J, Neckers LM, Haystead T, Spector NL, et al. Amplification and high-level expression of heat shock protein 90 marks aggressive phenotypes of human epidermal growth factor receptor 2 negative breast cancer. Breast Cancer Res. 2012;14:R62.

35. de la Mare JA, Jurgens T, Edkins AL. Extracellular Hsp90 and TGFbeta regulate adhesion, migration and anchorage independent growth in a paired colon cancer cell line model. BMC Cancer. 2017; 17:202.

36. Marzook H, Deivendran S, George B, Reshmi G, Santhoshkumar TR, Kumar R, et al. Cytoplasmic translocation of MTA1 coregulator promotes de-repression of SGK1 transcription in hypoxic cancer cells. Oncogene. 2017;36:5263-73.

37. Verba KA, Wang RY, Arakawa A, Liu Y, Shirouzu M, Yokoyama S, et al. Atomic structure of Hsp90-Cdc37-Cdk4 reveals that Hsp90 traps and stabilizes an unfolded kinase. Science. 2016;352:1542-7.

38. Li S, Li J, Hu T, Zhang C, Lv X, He S, et al. Bcl-2 overexpression contributes to laryngeal carcinoma cell survival by forming a complex with Hsp90beta. Oncol Rep. 2017;37:849-56.

39. Kim SH, Ji JH, Park KT, Lee JH, Kang KW, Park JH, et al. Highlevel expression of Hsp90beta is associated with poor survival in resectable non-small-cell lung cancer patients. Histopathology. 2015;67:509-19.

40. Xiong X, Wang Y, Liu C, Lu Q, Liu T, Chen G, et al. Heat shock protein 90beta stabilizes focal adhesion kinase and enhances cell migration and invasion in breast cancer cells. Exp Cell Res. 2014;326:78-89.

41. Correia AL, Mori H, Chen EI, Schmitt FC, Bissell MJ. The hemopexin domain of MMP3 is responsible for mammary 
epithelial invasion and morphogenesis through extracellular interaction with HSP90beta. Genes Dev. 2013;27:805-17.

42. Giessrigl B, Krieger S, Rosner M, Huttary N, Saiko P, Alami M, et al. Hsp90 stabilizes Cdc25A and counteracts heat shockmediated Cdc25A degradation and cell-cycle attenuation in pancreatic carcinoma cells. Hum Mol Genet. 2012;21:4615-27.

43. Didelot C, Lanneau D, Brunet M, Bouchot A, Cartier J, Jacquel A. et al. Interaction of heat-shock protein 90beta isoform (HSP90beta) with cellular inhibitor of apoptosis 1 (c-IAP1) is required for cell differentiation. Cell Death Differ. 2008;15:85966.

44. Sun Y, Huang YH, Huang FY, Mei WL, Liu Q, Wang CC, et al. 3'-epi-12beta-hydroxyfroside, a new cardenolide, induces cytoprotective autophagy via blocking the Hsp90/Akt/mTOR axis in lung cancer cells. Theranostics. 2018;8:2044-60.

45. Vinyoles M, Del VB, Curto J, Padilla M, Villarroel A, Yang J, et al. Activation of CK1varepsilon by PP2A/PR61varepsilon is required for the initiation of Wnt signaling. Oncogene. 2017;36:429-38.

46. Casagolda D, Del VB, Valls G, Lugilde E, Vinyoles M, CasadoVela J, et al. A p120-catenin-CK1epsilon complex regulates Wnt signaling. J Cell Sci. 2010;123:2621-31.

47. Knoblich K, Wang HX, Sharma C, Fletcher AL, Turley SJ, Hemler ME. Tetraspanin TSPAN12 regulates tumor growth and metastasis and inhibits beta-catenin degradation. Cell Mol Life Sci. 2014;71:1305-14

48. Lyou Y, Habowski AN, Chen GT, Waterman ML. Inhibition of nuclear Wnt signalling: challenges of an elusive target for cancer therapy. Br J Pharmacol.2017;174:4589-99.

49. Fu Y, Zheng S, An N, Athanasopoulos T, Popplewell L, Liang A, et al. Beta-catenin as a potential key target for tumor suppression. Int J Cancer. 2011;129:1541-51.

50. Zhao L, Li W, Zang W, Liu Z, Xu X, Yu H, et al. JMJD2B promotes epithelial-mesenchymal transition by cooperating with beta-catenin and enhances gastric cancer metastasis. Clin Cancer Res. 2013;19:6419-29.

51. Ha GH, Park JS, Breuer EK. TACC3 promotes epithelialmesenchymal transition (EMT) through the activation of PI3K/ Akt and ERK signaling pathways. Cancer Lett. 2013;332:63-73.

52. Zhang B, Yang Y, Shi X, Liao W, Chen M, Cheng AS, et al. Proton pump inhibitor pantoprazole abrogates adriamycinresistant gastric cancer cell invasiveness via suppression of Akt/ GSK-beta/beta-catenin signaling and epithelial-mesenchymal transition. Cancer Lett. 2015;356:704-12.
53. Yanaka Y, Muramatsu T, Uetake H, Kozaki K, Inazawa J. miR544a induces epithelial-mesenchymal transition through the activation of WNT signaling pathway in gastric cancer. Carcinogenesis. 2015;36:1363-71.

54. Zhao X, He L, Li T, Lu Y, Miao Y, Liang S, et al. SRF expedites metastasis and modulates the epithelial to mesenchymal transition by regulating miR-199a-5p expression in human gastric cancer. Cell Death Differ. 2014;21:1900-13.

55. Kannan A, Krishnan A, Ali M, Subramaniam S, Halagowder D, Sivasithamparam ND. Caveolin-1 promotes gastric cancer progression by up-regulating epithelial to mesenchymal transition by crosstalk of signalling mechanisms under hypoxic condition. Eur J Cancer. 2014;50:204-15.

56. Zhu XH, Wang JM, Yang SS, Wang FF, Hu JL, Xin SN, et al. Down-regulation of DAB2IP promotes colorectal cancer invasion and metastasis by translocating hnRNPK into nucleus to enhance the transcription of MMP2. Int J Cancer. 2017;141:172-83.

57. Gu Y, Wang Q, Guo K, Qin W, Liao W, Wang S, et al. TUSC3 promotes colorectal cancer progression and epithelialmesenchymal transition (EMT) through WNT/beta-catenin and MAPK signalling. J Pathol. 2016;239:60-71.

58. Yang SS, Li XM, Yang M, Ren XL, Hu JL, Zhu XH, et al. FMNL2 destabilises COMMD10 to activate NF-kappaB pathway in invasion and metastasis of colorectal cancer. $\mathrm{Br} \mathrm{J}$ Cancer. 2017; 117:1164-75.

59. Chen D, Liu G, Xu N, You X, Zhou H, Zhao X, et al. Knockdown of ARK5 expression suppresses invasion and metastasis of gastric cancer. Cell Physiol Biochem. 2017;42:1025-36.

60. Liang L, Li X, Zhang X, Lv Z, He G, Zhao W, et al. MicroRNA137, an HMGA1 target, suppresses colorectal cancer cell invasion and metastasis in mice by directly targeting FMNL2. Gastroenterology. 2013;144:624-635e4.

61. Xiao H, Liu Y, Liang P, Wang B, Tan H, Zhang Y, et al. TP53TG1 enhances cisplatin sensitivity of non-small cell lung cancer cells through regulating miR-18a/PTEN axis. Cell Biosci. 2018;8:23.

62. Singh A, Singh A, Sand JM, Bauer SJ, Hafeez BB, Meske L, et al. Topically applied Hsp90 inhibitor 17AAG inhibits UVR-induced cutaneous squamous cell carcinomas. J Invest Dermatol. 2015;135:1098-107.

63. Saveliev SV, Woodroofe CC, Sabat G, Adams CM, Klaubert D, Wood K, et al. Mass spectrometry compatible surfactant for optimized in-gel protein digestion. Anal Chem. 2013;85:907-14. 